\title{
1 Mechanism of transcription modulation by the transcription-repair coupling factor
}

2 Bishnu P Paudel ${ }^{1,2}$, Zhi-Qiang $\mathrm{Xu}^{1,2}$, Slobodan Jergic ${ }^{1,2}$, Aaron J Oakley ${ }^{1,2}$, Nischal Sharma ${ }^{1,2}$,

3 Simon HJ Brown ${ }^{1,2}$, James C Bouwer ${ }^{1,2}$, Peter J Lewis ${ }^{3}$, Nicholas E Dixon ${ }^{1,2}$, Antoine M van

4 Oijen $^{1,2}$, Harshad Ghodke ${ }^{1,2 *}$

$5{ }^{1}$ Molecular Horizons and School of Chemistry and Molecular Bioscience, University of Wollongong,

$6 \quad$ Wollongong, Australia

$7 \quad{ }^{2}$ Illawarra Health and Medical Research Institute, Wollongong, Australia

$8{ }^{3}$ School of Environmental and Life Sciences, University of Newcastle, Callaghan, NSW 2308, Australia

9 Correspondence:

10 Harshad Ghodke, Molecular Horizons and School of Chemistry and Molecular Bioscience, University

11 of Wollongong, Wollongong, New South Wales, 2522, Australia

12 


\section{Abstract}

15 Elongation by RNA polymerase is extensively modulated by accessory factors. The transcription-repair coupling factor (TRCF) recognizes distressed RNAPs and either rescues transcription or initiates

17 transcription termination. Precisely how TRCFs choose to execute either outcome remains unclear.

18 With Escherichia coli as a model, we used single-molecule assays to study dynamic modulation of 19 elongation by Mfd, the bacterial TRCF. We found that nucleotide-bound Mfd chaperones the 20 elongation complex (EC) into a catalytically active state, presenting the EC with an opportunity to

21 restart transcription. After long-lived residence in this catalytically poised state, ATP hydrolysis by Mfd

22 remodels the EC through an irreversible process leading to loss of the RNA transcript. Further, electron

23 cryo-microscopy revealed that the motor domain of Mfd binds and partially melts DNA containing a

24 template strand overhang. The results explain pathway choice determining the fate of the elongation 25 complex and provide a molecular mechanism for transcription modulation by TRCF. 


\section{Introduction}

Gene expression involves synthesis of RNA transcripts by RNA polymerases across all domains of life.

During elongation, RNA polymerase (RNAP) maintains a 9-10 nucleotide RNA:DNA hybrid within the

transcription bubble through the templated addition of ribonucleotides (reviewed by (Belogurov and

Artsimovitch, 2019)). Processive nucleotide addition cycles result in the growth of the RNA transcript

that is then extruded from the polymerase through the exit channel, accompanied by translocation of the polymerase along the template. Finally, during termination, nucleotide addition ceases and RNAP is displaced from the template DNA and recycled for subsequent rounds of transcription.

DNA template or at sites of roadblocks in the form of DNA-bound proteins. Prolonged residence in register with the 3' end of the RNA transcript, allowing the newly synthesized transcript to invade the secondary channel of RNAP (Abdelkareem et al., 2019; Gabizon et al., 2018; Komissarova and Kashlev, 1997). Rescue from such backtracked states is catalyzed by processivity factors such as GreA and GreB (Abdelkareem et al., 2019; Borukhov et al., 1993). Elongation is modulated by the universally conserved elongation/pause factor, the bacterial NusG, homologous with Spt5 in eukaryotes (reviewed by (Wang and Artsimovitch, 2020)). In Escherichia coli, NusG maintains processive transcription (Burova et al., 1995) by suppressing backtracking through contacts on the upstream DNA (Herbert et al., 2010; Kang et al., 2018). either transcription termination or rescue. The bacterial TRCF - Mfd is a multi-domain adaptor protein consisting of 7 domains connected by flexible linkers (Deaconescu et al., 2006). Domains $1 \mathrm{a} / 2 / 1 \mathrm{~b}$ form the UvrB-homology module that bears structural homology to the nucleotide excision repair protein 

(Kang et al., 2021). Domains 5 and 6 together form the motor domain, representing a superfamily II ATPase that exhibits structural homology to the RecG translocase (Mahdi et al., 2003). Domain 7 is an auto-inhibitory domain that contacts the UvrB-homology module to maintain it in a closed conformation, suppressing promiscuous DNA binding of Mfd in the absence of interactions with RNAP. structural methods (Brugger et al., 2020; Deaconescu et al., 2006; Kang et al., 2021; Shi et al., 2020), as well as single-molecule (Fan et al., 2016; Graves et al., 2015; Ho Wan et al., 2012; Le et al., 2017) and live-cell imaging techniques (Ghodke et al., 2020; Ho et al., 2018, 2020), a picture has been formed of the bacterial TRCF Mfd acting in a complex role in modulating transcription activity, including at hard-to-transcribe regions (Ragheb et al., 2021). At sites of DNA damage, Mfd orchestrates premature transcription termination leading to an enhancement in repair of DNA damage in the template strand - a phenomenon known as transcription-coupled DNA repair (Fan et al., 2016; Ghodke et al., 2020; Haines et al., 2014; Ho et al., 2020; Mellon and Hanawalt, 1989; Selby and Sancar, 1993a). In bacterial cells, the UvrA ${ }_{2} B$ nucleotide excision repair machinery is recruited to the site of distressed RNAPs by Mfd, resulting in the site-specific loading of the damage verification enzyme UvrB (Ho et al., 2020). This ability to recruit DNA-repair machinery at sites of lesion-stalled RNAP to promote ATP-dependent premature transcription termination is a property that is shared by eukaryotic functional homologs of Mfd - the yeast Rad26 (Duan et al., 2020; Xu et al., 2017) and human CSB (van der Weegen et al., 2020). Mutations in CSB protein result in deficient repair machinery recruitment causing Cockayne syndrome in humans (Mayne and Lehmann, 1982; Troelstra et al., 1992; Venema et al., 1990). elongation factors. Like Rad26 (Xu et al., 2017) and CSB (Selby and Sancar, 1997), Mfd has also been shown to rescue transcription at pause sites in vitro through a 'release-and-catch up' mechanism (Le et al., 2017; Park et al., 2002). Forward translocation of the elongation complex (EC) is central to TRCF 
activities including rescue from backtracked states, and transcription termination (Le et al., 2017; Park et al., 2002; Selby and Sancar, 1997; Xu et al., 2017). Considering their membership in the class of superfamily 2 helicases (Byrd and Raney, 2012), the structural homology of the TRCF motor domains and a similar mode of engagement with the elongation complex (EC) between Mfd (Brugger et al., 2020; Kang et al., 2021; Shi et al., 2020) and Rad26 (Xu et al., 2017), it is plausible that the molecular mechanisms underlying these outcomes are conserved. models have been proposed to explain forward translocation of RNAP by TRCFs: first, the TRCF 'pushes' RNAP forward while translocating on dsDNA as proposed for Mfd (Park et al., 2002), and second, the TRCF pulls the ssDNA template to effectively translocate the polymerase forward (Xu et al., 2017). Recent structures of EcMfd approaching an in vitro reconstituted EC assembled on a noncomplementary bubble have demonstrated that following initial recognition of the EC, Mfd translocates forward until it bumps into the RNAP, pushing the polymerase and resulting in global remodeling of the EC (Kang et al., 2021). The structure of yeast Rad26 bound to upstream DNA in the RNA Pol II EC (Xu et al., 2017) reveals direct contacts between dsDNA-bound Rad26 and ssDNA template strand. Based on the homology to the Swi2/Snf2-family core ATPase domains, and the mechanism of Snf2 in chromatin remodeling, it has been proposed that Rad26 pulls template DNA in the Pol II EC (Xu et al., 2017). Structures of DNA-bound Mfd have established the homology of its motor domains to ATPases involved in chromatin remodeling (most notably Snf2) (Brugger et al., 2020). Since available structures depict initial global remodeling of the EC but not transcription termination leading to disassembly of the EC accompanied by loss of the transcript (Kang et al., 2021), it remains to be seen whether a similar Mfd-ssDNA complex forms during transcription termination.

100 Further, while it is clear that displacement of the polymerase in the absence of downstream binding 101 partners of $\mathrm{Mfd}$ is a slow event requiring several minutes in vitro (Graves et al., 2015; Ho Wan et al., 102 2012), the sequence of events leading to loss of the transcript, and its residence time in the EC during 103 this remodeling remains unclear. 

of the RNA transcript under various scenarios. We developed a single-molecule Förster resonance energy transfer (smFRET)-based assay that directly visualizes dynamic changes in the distance between donor-labeled upstream DNA and acceptor-labelled RNA transcript forming the transcription bubble. We reconstituted catalytically active ECs in situ and monitored the FRET signal from surfaceimmobilized molecules under conditions of partial or complete nucleotide starvation, and probed global remodeling of the EC by NusG and Mfd on the timescale of minutes. NusG efficiently rescued

Mfd was able to rescue ECs from catalytically inactive states into a long-lived catalytically poised state,

113 prior to continued ATP-hydrolysis dependent forward translocation consistent with transcription

114 termination. This catalytically poised state acts as a 'molecular timer', presenting the EC with an 115 opportunity to restart transcription. Further biophysical and structural biology studies collectively reveal that in addition to the previously described dsDNA binding mode, Mfd makes contacts with ssDNA on DNA substrates mimicking the transcription bubble.

\section{Rationale for a smFRET assay to study transcription elongation}

We monitored transcription elongation by assembling functional ECs on dye-labeled fullycomplementary transcription bubbles in situ by adopting a previously described strategy to study RNA exhibit high smFRET signals within the dynamic range of the technique. Three features of this strategy enable precise dissection of the dynamics of molecular mechanisms of Mfd-dependent remodeling of

127 the EC: (i) the use of synthetic RNA and DNA nucleotides enables site-specific introduction of dyes in 128 the phosphodiester backbone that serve as FRET pairs, and along with it, the ability to probe conformational changes between various parts of this complex (ii) smFRET affords sub-second 
temporal resolution $(200 \mathrm{~ms})$ enabling reliable measurements of dynamic interactions on the timescale of seconds to minutes, and (iii) the ability to specify RNA sequences that cannot hybridize with upstream DNA limits extensive backtracking of ECs. RNAP in the elongation phase can exist as a backtracked complex (where the $3^{\prime}$ end of the transcript is extruded into the secondary channel

134 (Abdelkareem et al., 2019)), a pre-translocated conformation prior to nucleic acid addition, and a posttranslocated conformation following nucleic acid incorporation. Since backtracking requires sequence is expected to minimize the heterogeneity in the observed population of ECs in this assay.

Monitoring transcription elongation by RNAP

140 been pre-incubated with the template:RNA hybrid into a microfluidic device with surface immobilized

141 biotinylated non-template DNA. After complex assembly, the flow cell was washed with a high-ionic

142 strength solution $(1 \mathrm{M} \mathrm{KCl})$ followed by rapid exchange with a buffer solution supporting transcription but without ribonucleotides (NTPs). Subsequent measurements of the smFRET signal between the Cy3 donor and the Cy5 acceptor (see Methods) reveals complexes assembled resided in stable high FRET states centered at 0.9 (Figure 1B upper trace). In addition, a small fraction (15\% of 318 trajectories) also exhibited mid-FRET states centered at 0.6 (Figure 1B lower trace). These states were recapitulated

147 in temporal heatmaps (Figure 1C) that are scatterplots describing how the smFRET signal evolves in

148 time for the observed population. These temporal heat maps are a convenient representation 149 presenting an overview of the progress of FRET trajectories over time. Here, the overlaid black lines 150 reflect contours parsing the data into deciles.

151 Since the majority of the complexes thus assembled exhibited time-FRET trajectories that

152 fluctuate around $0.9 \mathrm{FRET}$, and a minority exhibited transitions to the 0.6 FRET state, we pursued an 153 unbiased approach to visualize transitions between various FRET states. FRET trajectories were 154 idealized using vbFRET (http://vbfret.sourceforge.net/) and the detected states were plotted in 
among different states and the directionality of those changes (Bronson et al., 2009). In this representation, features that are only present in one half of the plot on either side of the diagonal manifest as asymmetric features and denote irreversible transitions between states on the timescale of the observation. As demonstrated by the TDP, the interconversions between the states were reversible, and were restricted to the high FRET regime. Together, the observed symmetry of these peaks and their proximity to the diagonal, and to each other indicate that the complex thus reconstituted transitions between states reversibly. Within the paradigm that FRET changes reflect conformational changes, a simple interpretation of these data is that the complex undergoes relatively minor, but reversible, conformational changes. For convenience, here we assign smFRET signals between $0.7-1$ as being 'high', $0.4-0.7$ as 'mid' and $0-0.4$ as being 'low'.

To assign states, and to test that the complexes are catalytically functional, we challenged complexes with deoxyNTPs (dNTPs), 'wrong' (or non-complementary) NTPs and the complete set of NTPs. In this assay, transcript elongation is expected to increase the distance between the FRET pair dictated by the geometry of the EC, leading to a drop in FRET. Since dNTPs are selected against incorporation into the transcript (Svetlov et al., 2004), we expected that SmFRET signal from the reconstituted ECs would not drop. Consistent with this expectation, complexes exposed to dNTPs revealed a high-FRET population (Figure 1D, 1E). In contrast, non-complementary NTPs converted the population of complexes in the high-FRET states to the mid-FRET states (Figure 1F, G). In both cases, the interconverting populations were found to be symmetric across the diagonal indicating that in the presence of either dNTPs or the wrong NTPs, the ternary complex visits the observed states reversibly (Supplemental Figure 1A).

'Walking' the complex by supplying a subset of the 'correct' complementary NTPs revealed 178 gradual conversion from the high-FRET to a mid-FRET population (Supplemental Figure 1B-D). When 179 presented with the complete set of NTPs, the ternary complex exhibited unidirectional conversion of the FRET signal from high- to low-FRET values (Figure 1H, 1 l and Supplemental Figure 1A). Notably, the transition density plots, and indeed the individual traces, revealed a conversion of the high-FRET 
states to the low-FRET states via the mid-FRET states.

183 EC during the nucleotide addition cycle.

\section{NusG promotes residence of ECs in the catalytically poised state} restricts conformational rearrangements of the EC.

Collectively, the data indicate that the complexes assembled in situ represent functional ternary ECs that exist in a catalytically 'inactive' state and a catalytically 'poised' state. The observations that complexes first transition to the mid-FRET state (0.6) prior upon addition of NTPs suggests that this represents a catalytically poised state. We tentatively assigned the high-FRET states centered at 0.9 to a catalytically inactive state. This catalytically inactive state may represent a pretranslocated or backtracked conformation of the EC. Considering that the sequence of the $5^{\prime}$ end of the transcript is non-complementary to the upstream DNA, we expect that extensive backtracking is disfavored on this substrate. We propose that the likeliest explanation is that the high- and mid- FRET states detected here represent pre- and catalytically poised post-translocated states accessed by the

Next, we tested this assignment of states by assessing the response of the complex to the elongation factor-EcNusG (Figure 2A), which is predicted to retain ECs in the catalytically poised state. Addition of NusG indeed resulted in an increased occupancy of mid-FRET states accompanied by a reduction of the high-FRET (Figures 1B, 1C cf. 2B, 2C). Trajectories revealed a conversion from the high-FRET state into the mid-FRET state and fluctuation around the mid-FRET state (Figure 2B). This behavior confirms our assignment of the mid-FRET state as the catalytically active state. The reverse transition from midFRET to high-FRET states that would reflect unbinding of NusG appears to be disfavored in this experimental condition as the detected asymmetric features in the transition density plot are unidirectional (Supplemental Figure 2A). These trajectories are readily explained if binding of NusG

Incubation with incorrect NTPs in the presence of NusG revealed enrichment of molecules exhibiting the 0.6 FRET state (Figure 2E, 2F), similar to that observed in the case of incorrect NTPs alone (Figure 1F, 1G). Reversible short-lived transitions to low-FRET states were also evident in this data set (Figure 2D, 2E and Supplemental Figure 2A). These transitions may reflect changes in the path 
of the upstream DNA that have been detected previously (Kang et al., 2018). Importantly, the complete set of NTPs revealed efficient, irreversible conversion to the low-FRET states $(0-0.2)$ from the mid-FRET state (Figure 2F, 2G and Supplemental Figure 2A) in the presence of NusG suggesting processive extension of the transcript through catalysis and associated forward translocation by RNAP. represent inactive states.

\section{Remodeling of the elongation complex by Mfd}

218 With the assignment of the high-FRET state as a catalytically inactive state, and the mid-FRET state as

219 a catalytically poised state, we next assessed the interaction of Mfd with ECs. To characterize the binding of Mfd to RNAP, we first measured the affinity of Mfd for pre-assembled ECs, or the dsDNA alone using surface plasmon resonance (SPR). Since Mfd interacts poorly with DNA in bulk assays in the absence or presence of ATP, measurements of binding of purified Mfd to either DNA or the EC were carried out in the presence of slowly hydrolyzable ATP $\gamma$ S. SPR measurements revealed a dissociation constant $\left(K_{\mathrm{D}}\right)$ of $170 \pm 20 \mathrm{nM}$ (Supplemental Figure 2B) for 49-mer dsDNA alone, consistent with recent measurements by other groups (Brugger et al., 2020; Le et al., 2017). Binding

Figure 2C). These data are consistent with the model that dsDNA serves as a major binding partner for Mfd-ATP $\gamma \mathrm{S}$ in the context of the EC as previously demonstrated (Kang et al., 2021; Le et al., 2017; Park et al., 2002; Shi et al., 2020).

We then investigated how interactions of Mfd with RNAP influence the conformational dynamics of the transcription bubble using our smFRET assay (Figure 3A). Consistent with the lack of stable binding of Mfd with DNA in the absence of ATP, Mfd engaged the EC poorly in the smFRET measurements with $58 \%$ of trajectories ( $n_{\text {obs }}=75$ ) presenting no observable interactions with Mfd 
234 (Figure 3B lower panel). The remaining trajectories exhibited reversible transitions from the high- to

235 mid-FRET states even in the absence of any nucleotide co-factor (Figure 3B upper panel, 3C and Supplemental Figure 3A 'RNAP, Mfd') reflecting attempts at stable engagement of Mfd with the EC. Notably, differently from the EC alone (Supplemental Figure 1A), dynamic trajectories exhibited transitions mainly in the mid-FRET region (Figure 3B upper panel, Supplemental Figure 3A 'RNAP, Mfd').

Mfd-ATP $\gamma$ S resulted in dynamic ECs visiting additional mid FRET states (Figure 3D, 3E and

241 Supplemental Figure 3A 'RNAP, Mfd, ATP $\gamma S^{\prime}$; 59\% of 69 trajectories presented dynamic behavior).

242 ATP $\gamma$ S alone did not account for these observations (Supplemental Figure 3B). Curiously, Mfd-ATP $\gamma$ S exhibits quantized transitions in the transition density plot compared to Mfd alone, indicating that binding of Mfd-ATP $\gamma S$ displaces RNAP forward (Supplemental Figure 3A 'RNAP, Mfd' vs. 'RNAP, Mfd, ATP $\left.\gamma S^{\prime}\right)$. Together the data reveal that Mfd can isomerize RNAP merely upon binding to the nucleotide starved EC. The efficiency of this isomerization is appreciably greater in the presence of ATP $\gamma$ S. trajectories in the data set: (i) static trajectories that exhibited high FRET values throughout the observation reflecting molecules that are presumably unaffected by Mfd-ATP (54/176 observations; 31\%), (ii) dynamic trajectories exhibiting high FRET values at the start of the observation resulting in a direct transition to low-FRET states (24/176 trajectories, 14\%; Figure 3F lower panel), (iii) dynamic trajectories starting at a high-FRET value but transiting to a low-FRET state through a single mid-FRET intermediate (56/176 trajectories; 32\%, Figure 3F upper panel) and (iv) dynamic trajectories that are at the mid-FRET state at the start of the observation ( $42 / 176$ corresponding to $24 \%$; compared to $14 \%$ of observations in the absence of Mfd). Since the dataset presented here represents a pooled data set from several videos collected after Mfd-ATP was introduced to the flow chamber, this last category of trajectories likely reflects ECs that are already being remodeled by Mfd at the start of the observation. 
individual ECs (Figure 3F, upper panel). The intermediate step was randomly distributed in the range of FRET from 0.35 to 0.5 (Supplemental Figure 3C). Strikingly, the lifetime of this mid-FRET state did not exhibit a dependence on the concentration of ATP in the range from $10 \mu \mathrm{M}$ to $10 \mathrm{mM}$ (Supplemental Figure 3D). To further explore the nature of these transitions, we visualized them in a transition density plot (Supplemental Figure 3A, 'RNAP, Mfd, ATP'; entire data set is plotted here). Notably, irreversible transitions manifesting as asymmetric features in these plots appeared mainly as transitions from the mid-FRET to low-FRET states (Supplemental Figure 3A, 'RNAP, Mfd, ATP', red ellipse). As a control, titration of ATP in the absence of Mfd resulted in RNAP complexes exhibiting primarily a high-FRET population, with a gradual increase in the mid-FRET state at millimolar concentrations (Supplemental Figure 3E). Compared to the wild-type, the $\mathrm{Mfd}(\mathrm{R} 953 \mathrm{~A})$ mutant (Chambers et al., 2003), known to be proficient in ATP hydrolysis while strikingly deficient in RNAP displacement and transcription termination exhibited inefficient conversion to the low-FRET states (Figure 3H, 3I and Supplemental Figure 3A 'RNAP, Mfd(R953A), ATP'). Notably, unlike wild-type Mfd, irreversible transitions were observed mainly in the high- to mid- FRET regime (Supplemental Figure 3A 'RNAP, Mfd(R953A), ATP', red ellipse). Intriguingly, Mfd(R953A)-ATP exhibited a pattern of quantized transitions that is similar to that of Mfd-ATP $\gamma \mathrm{S}$ in the transition density plots (Supplemental Figure 3A, compare 'RNAP, Mfd, ATP $\gamma S^{\prime}$ ' and 'RNAP, $M f d(R 953 A)$, ATP'), suggesting that the mutation in this residue serves to reveal distinct aspects of Mfd's binding to the EC. Together, the data indicate that both wild-type and translocase deficient Mfd rescue RNAP 279 from the catalytically inactive (potentially pre-translocated or weakly backtracked) states accessible in our substrate to catalytically-poised states, albeit with varying efficiencies. However, only the wildtype Mfd can further process the EC in the presence of ATP. The low-FRET states observed in our system represent circumstances in which the distance between the donor-acceptor pair is outside the dynamic range of efficient FRET as might be expected when the RNAP is forward translocated well past the stall site and/or the Cy5-labelled RNA transcript is lost. The observation that the EC fails to return to the mid- or high-FRET states rules out a scenario where this low-FRET state simply reflects 
an over-wound transcription bubble, or a bent upstream DNA conformation as is seen in the case of NusG (Kang et al., 2018). Hence, this loss of FRET is most consistent with an irreversible loss of RNA. In this model, Mfd(R953A) fails to displace the transcript from the $\mathrm{EC}$, consistent with prior findings (Chambers et al., 2003).

\section{Mfd contacts template ssDNA}

Structures of Mfd bound to dsDNA and ECs containing non-complementary transcription bubbles have revealed a series of translocation intermediates that are formed prior to and leading up to stable engagement of Mfd and initial disruption of the EC (Kang et al., 2021; Shi et al., 2020). Currently, little is known about the intermediate states that follow this initial contact. We set out to identify whether Mfd makes contacts with ssDNA in substrates mimicking the upstream edge of the transcription bubble by employing a combination of approaches: First, we employed smFRET experiments to detect whether and how Mfd contacts template ssDNA. Second, we visualized Mfd bound to a primed DNA template using cryo-EM.

To detect ATP-dependent contacts of Mfd with ssDNA we employed a previously described smFRET assay to study translocase activity (Park et al., 2010). We used a DNA substrate comprised of an 18-mer dsDNA duplex (mimicking upstream DNA) and a $5^{\prime} \mathrm{dT}_{40}$ overhang mimicking the template DNA (Figure 4A) with a FRET pair positioned on either end of the ssDNA overhang (Table 1: bio_AS18_Cy5: Cy3_dT40_S18, see Materials and Methods). In smFRET experiments, this substrate exhibited an average FRET of $0.33 \pm 0.15$ (Figure 4B) in the absence of Mfd reflecting rapid sampling of various conformations accessed by the entropic coil formed by ssDNA (Figure $4 C$ and Supplemental Figure 4A 'template overhang $\mathrm{DNA}^{\prime}$ ). Mfd binds this substrate with a $K_{\mathrm{D}}$ of $390 \pm 10 \mathrm{nM}$ (see

307 Supplemental Information and Supplemental Figure 4B-D).

In the presence of ATP $\gamma \mathrm{S}$, the smFRET signal from the Mfd-bound substrate revealed additional distinct populations at higher FRET values of 0.6 and 0.8 (Figure 4D, 4E and Supplemental Figure 4A 'Mfd, ATP $\gamma S^{\prime}$ '). Transitions among these states were further enhanced in the presence of ATP

311 as the substrate exhibited a continuous spectrum of mid- and high-FRET states (Figure 4F, 4G and 
312 Supplemental Figure $4 \mathrm{~A}$ ' $\mathrm{Mfd}, \mathrm{ATP}$ ') in addition to the distinct states detected in the presence of

313 ATP $\gamma$ S. In this case, the higher FRET states were only transiently accessed indicating that ATP hydrolysis

314 plays a role in exiting from these high-FRET states to low-FRET states. This observation is consistent with a picture in which Mfd-ATP contacts the ssDNA to bring the dyes close to each other resulting in a high FRET signal, and subsequent ATP hydrolysis induces a lower-affinity conformation in which Mfd-ADP binds the ssDNA very weakly resulting in a return to the baseline FRET value of 0.33 . (Deaconescu et al., 2012; Ho et al., 2018), this template exhibited high-FRET states extremely rarely compared to the wild-type protein (Supplemental Figure 4F, 4G). Therefore, the observation that Mfd(E730Q)-ATP is unable to stably engage with ssDNA, unlike Mfd-ATP $\gamma$ S suggests that the Mfd(E730Q)-ATP and Mfd-ATP $\gamma$ S pre-hydrolytic states are sufficiently different that only the wild-type Mfd retains the ability to engage ssDNA efficiently. engage ssDNA on primed DNA templates (Figure 4H, 4I). Therefore, we conclude that both the ability 327 to hydrolyze bound ATP and the presence of an intact R953 are essential for Mfd to engage the ssDNA 328 template DNA overhang. We propose that R953 serves to 'hook' ssDNA, and ATP binding serves to 329 'pull' ssDNA closer to the junction resulting in the high FRET signal detected in this assay with wildtype Mfd. We note that the recent structures of Mfd translocating on dsDNA indicate that R953 along with key residues in the translocation motif (TRG: translocation in RecG (Mahdi et al., 2003)) of Mfd, specifically R929 and Q963, contacts the non-template strand in dsDNA. From these structures it is not immediately obvious how the template strand overhang is contacted by Mfd in the smFRET assays.

334 The results presented here uncover a new role for R953 in making critical contacts with the template ssDNA distinct from its previously described role in translocation on dsDNA. 
immediately, Mfd (or mutants) in the presence of ATP entered this state in a time window $\sim 5-20$ seconds after introduction into the flow cell (Figure 4E, 4G, $4 \mathrm{I}$ and Supplemental Figure 4H). The presence of the low-FRET peak and the excursions to high-FRET states suggested that multiple independent events may underlie the FRET signal observed here. Whereas all conditions involving Mfd

342 (or mutant) exhibited the low-FRET peak, the excursions to the high FRET states were only observed

343 for wild type Mfd in the presence of ATP or non-hydrolyzable analog.

\section{Mfd melts dsDNA upon contacting template ssDNA}

To further understand how Mfd binds to 5' overhang-containing DNA, we visualized Mfd bound to the dye labelled smFRET substrate by cryo-EM (Table 1: bio_AS18_Cy5: Cy3_dT40_S18, see Materials and Methods). Full-length Mfd bound to the primed-template substrate in the presence of transition-state analog $A D P-A_{1} F_{x}$ was subjected to single particle cryo-EM analysis (Figure $5 A$ ) and a $\sim 5.2 \AA$ resolution map was obtained (real resolution $\sim 8 \AA$, Supplemental Figure $5 \mathrm{~A}-\mathrm{C}$ ). This reconstruction yielded electron density that could at best accommodate the motor domains of Mfd not be seen in the electron density maps. motor domains and the placement of the motor domains with respect to dsDNA is consistent with previously reported complexes (Brugger et al., 2020; Kang et al., 2021) (Figure 5B). However, density for residues 917 onward, containing the TRG motif, appeared to be absent from the side of domains

3575 and 6 , and the density of the DNA as it passes from the upstream side to the downstream side becomes inconsistent with dsDNA (Figure 5B). Using molecular dynamics flexible fitting (Singharoy et al., 2016) to equilibrate the structure in density suggests that the TRG motif has dropped into the path of the dsDNA (yellow ribbon, Figure $5 C$ ), resulting in the partial melting of the duplex. The motif forms an arch that interacts with the template DNA (Figure 5C, 5D). There are precedents for conformational 
ethanesulfonic acid bound between the helices, and are parallel in the complex with ADP-AlFx and dsDNA (PDB 6XEO). These movements are attributable to both to shifts in domains 5 and 6 relative to each other and to movements of the helices relative to each other. The conformation of domains 5 and 6 suggested here may represent a mode of engagement whereby Mfd grabs and stabilizes the template DNA in the upstream edge of the transcription bubble. ssDNA three and six nucleotides away from the junction respectively (Figure $5 C, 5 F)$. ADP-AlF - -bound Mfd scrunches the template ssDNA (Figure 5D-F), explaining the basis of the high-FRET signal observed in the smFRET experiments (Figure 4). The proximity of R953 to the ssDNA in the model also explains how R953 can hook the template ssDNA in an ATP-dependent manner (Figure 5C, 5F) and in case of the distressed EC, transform the energy of ATP binding and hydrolysis into forward translocation of RNAP. conserved basic residues in domain 5 (R685, K690, H711, K712, H732, R733, R737, K739, R761) (Figure 5G, blue spheres) along with polar (T710) (Figure 5G, yellow spheres) and aromatic (F734) (Figure 5G,

384 green spheres) residues comprise the surface in contact with the non-template DNA strand (Figure 385 5E). The binding mode proposed here is partially validated by prior observations implicating several 386 key conserved residues in domain 6 (Y816, N817, H842, T868 and R887) as well as domain 5 (R685, K690, T710, H711 and K712) implicated in DNA binding (Brugger et al., 2020; Le et al., 2017). Consistent 

edge of the transcription bubble: the cryo-EM data suggest that Mfd binds and partially melts the dsDNA near the junction, contacting the unpaired template ssDNA via the conserved R929 and R953 residues in the TRG motif, and the smFRET experiments reveal that Mfd dynamically cycles between high- and low-affinity states in an ATP-hydrolysis dependent manner on the template ssDNA overhang, pulling and scrunching the template strand in this process.

\section{Discussion}

In this work, we established that a 'molecular timer' intermediate formed by Mfd and the transcription elongation complex determines the fate of the elongation complex. Our attempts to understand this intermediate resulted in the discovery of a novel DNA-binding mode in which Mfd binds ssDNA in a transcription bubble mimic. Together, the results provide unprecedented insight into the transcription termination reaction and enable us to propose a comprehensive model describing how events unfold after initial engagement of the elongation complex with the transcription-repair coupling factor.

proceeds via multiple stages: In the first stage, Mfd recognizes a distressed polymerase and is recruited to the EC (Ho et al., 2018; Ho Wan et al., 2012; Le et al., 2017; Park et al., 2002; Proshkin and

411 reactivates paused transcription (Le et al., 2017; Park et al., 2002), or terminates transcription (Ho

412 Wan et al., 2012; Selby and Sancar, 1993a). Alongside these activities, Mfd recruits its binding partners

$413 \quad \mathrm{UvrA}_{2} \mathrm{~B}$ to the site during transcription-coupled DNA repair (Fan et al., 2016; Ghodke et al., 2020; Ho et al., 2018, 2020; Selby and Sancar, 1993a). 
reaction and present a new model for resolution of a critical intermediate. The SMFRET-based elongation modulation assay revealed that Mfd can engage the EC in an ATP independent manner and manifest transient, reversible conformational changes in the EC. Stable association requires ATP or at minimum non-hydrolysable ATP, likely required to impart a dsDNA binding conformation in the motor domain. Indeed, a recent study reported the structural basis of ATP-dependent translocation of Mfd following initial recognition of distressed elongation complexes leading to docking of Mfd with the upstream edge of the transcription bubble (Kang et al., 2021). Our findings agree well with these findings (Figure $6 \mathrm{~A}-\mathrm{C}$ ). states, Mfd-ATP, Mfd-ATP $\gamma$ S and Mfd(R953A)-ATP each chaperone the EC into a single long-lived gating intermediate (Figures 3 and 6D) hovering in the mid-FRET range controlling the transition to low-FRET states. Efficient entry into this state is granted to Mfd in the presence of ATP or ATP $\gamma$, whereas Mfd(R953A)-ATP enters this state only inefficiently. The observation that Mfd-ATP $\gamma \mathrm{S}$ is sufficient to promote entry into this state indicates that binding of ATP alone is a sufficient requirement for the formation of the gating intermediate.

The suggestion that Mfd-ADP-AlF 4 might bind and melt primed DNA templates to unwind dsDNA (Figure 5), provides insight into this transition. Only 13 nucleotides of the 18-mer DNA template are consistent with dsDNA, with the remainder being partially melted. The lack of observations of dsDNA melting in a recently reported structure (Brugger et al., 2020) suggests that Mfd requires access to unpaired ssDNA via critical residues in the TRG motif to melt the dsDNA. bound - Mfd partially melts about five nucleotides of dsDNA to produce a bubble, henceforth referred to as the 'TRCF bubble' (Figure 5). Considering the three nucleotide proximity of the binding site of Mfd to the upstream edge of the transcription bubble (Kang et al., 2021), the upstream DNA may further melt allowing the TRCF bubble to fuse with the transcription bubble to produce the isomeric 
442 lacking an overhang, a more likely possibility is that Mfd merely extends the transcription bubble by

443 further melting the upstream edge, resulting in essentially a TRCF-T bubble. formation of the TRCF-T bubble described here. overhang (Figure 5). The observation that Mfd(R953A)-ATP fails to stably engage the ssDNA overhang in our smFRET study supports a model in which this residue (potentially along with other residues in

461 the translocation motif) serves to stabilize the fork at the downstream edge of the TRCF bubble, or 462 template ssDNA in the TRCF-T bubble. We propose that binding of R953 to the ssDNA template occurs 463 simultaneously with the melting of the upstream dsDNA. Evidently along with a role in DNA translocation, R953 has a newly discovered role in hooking and stabilizing ssDNA. Our structure also predicts a critical role for R929 in this activity. 
a contact through R953. Although available structures of Mfd-[ATP or non-hydrolyzable analog]-EC do not report melting of the upstream DNA or a TRCF-T bubble, and a structure of Mfd-EC bound to the TRCF-T bubble is not as yet forthcoming, precisely such an intermediate has been observed in the Rad26-Pol II structure where an extended TRCF-T bubble of 17 nucleotides is observed (Xu et al., 2017). The conserved motor domains of Rad26 contact three nucleotides each from partially unwound upstream DNA in this structure. Analogous to R953 in Mfd, R613 in Rad26 makes a contact with ssDNA in the TRCF-T bubble. These similarities between the prototypical members of this class of transcription-coupled DNA repair factors hint to a more profound conservation of mechanism of action. $\mathrm{EC}$ (or at minimum restricts backtracking of the EC) leading to the formation of the catalytically poised state of the RNAP. Here, in the presence of the correct NTP and an intact R953, resumption of transcription would lead to faster forward translocation of RNAP leaving behind Mfd-ATP bound to the TRCF bubble (Figure 6E). In effect this finding explains how Mfd can 'release' distressed polymerases in the so-called 'release and catch-up' model for transcription rescue (Le et al., 2017). A prediction of our model is that Mfd bound to a non-hydrolyzable ATP analog should be sufficient to induce transcription rescue. consider that exit from this intermediate is signified by a loss of FRET. To what might this irreversible loss of FRET correspond? This catalytically poised intermediate formed prior to irreversible remodeling of the EC is directly comparable to the Mfd-concentration independent intermediate detected in nanomanipulation assays (Ho Wan et al., 2012). However, two key differences are immediately apparent: first, whereas the lifetimes of the intermediate detected in our FRET studies follows a single exponential distribution with a lifetime of $6 \mathrm{~s}$ (reflecting a process with a single dominant rate-limiting step), the lifetimes of the intermediate observed in the previous study exhibit a Gaussian distribution 493 (reflecting a stepwise process with several comparable reaction rates). Second, the lifetime of the 
intermediate in the nanomanipulation assays $(335 \pm 3 s)$ is much longer than the lifetime of the intermediate detected here $(6 \mathrm{~s})$. To reconcile these differences, we note that the nanomanipulation assays reflect the extension of the supercoiled DNA as a proxy for rewinding of the transcription bubble, while our smFRET measurements reflect the distance between the dye pair positioned on the upstream DNA and the RNA transcript. Thus loss of FRET observed once the complex exits from the gating intermediate can reflect either loss of the transcript, or forward translocation of the polymerase to a point where the acceptor is no longer within the dynamic range of the technique. Since the lifetime of the intermediate formed during complete rewinding of the transcription bubble ( $335 \mathrm{~s})$ (Ho Wan et al., 2012) is much longer than measured here, the irreversible process detected here likely describes loss of the RNA transcript (Figure 6D, F). This interpretation is supported by previous observations that loss of RNA from the EC occurs rapidly after Mfd binds the EC (Graves et al., 2015). is available beyond which, transcription restart is no longer possible. The prolonged residence in the catalytically poised state prior to irreversible remodeling of the EC by Mfd may serve to provide an opportunity to restart transcription at sites of pausing. This observation explains how Mfd can orchestrate transcription restart (Le et al., 2017) or termination (Ho Wan et al., 2012) via a kinetic proofreading mechanism (Hopfield, 1974) whereby paused ECs can be substrates for transcription restart, and irreversibly or DNA damage-stalled ECs are substrates for transcription termination. In effect, this gating intermediate serves as a molecular timer determining the fate of the EC.

514 proceeds via at least partial, and potentially complete, shrinking of the TRCF-T bubble leaving behind 515 Mfd bound to the TRCF bubble. How might these events play out? Our results demonstrate that the 516 lifetime of this state is only $6 \mathrm{~s}$ at $20^{\circ} \mathrm{C}$. While entry into this state is dependent on the presence of 517 ATP, the observation that this lifetime is independent of ATP concentration in the range from $10 \mu \mathrm{M}$ 518 to $10 \mathrm{mM}$, suggests that hydrolysis of stoichiometric amounts of ATP, may be sufficient to promote exit from this state. Further, an intact R953 is required for exit from this state. Since dissociation of 
the 9-nt RNA:DNA hybrid (possessing a melting temperature of $15^{\circ} \mathrm{C}$ ) is expected to be spontaneous at this temperature in the absence of stabilizing interactions with the EC, the loss of the transcript signals the resolution of the molecular timer, accompanied by the formation of an intermediate where RNAP is extensively remodeled, and the TRCF-T bubble is partially or completely rewound. The cryoEM derived model presented here sheds light on how Mfd may scrunch template DNA in this process. the polymerase, and R953 hooks the template ssDNA. Based on the structures of translocation intermediates of Mfd (Kang et al., 2021), we propose that ATP hydrolysis leads to unhooking of ssDNA

530 (Figure 4), completing the pull stroke. This in turn leads to re-zipping of the upstream edge of the TRCF 531 bubble and advancement of the TRCF bubble toward the EC. This ATP hydrolysis event triggers catastrophic remodeling of the EC (Figure 3 and Supplemental Figure 3), exposing the template-RNA (potentially through a rearrangement of the $\beta^{\prime}$ lid of RNAP) and enabling its rapid dissociation from the former EC. While this model awaits further validation, a prediction is that Mfd must possess an annealase activity as has been documented for the human homolog CSB (Muftuoglu et al., 2006). suggest that the transcription bubble shrinks by two-thirds of its size (Graves et al., 2015; Ho Wan et al., 2012). The observation that the transcription bubble was rapidly rewound upon interaction with

540 Mfd led the authors to suggest that this residual DNA extension reflects "bending/wrapping 541 interactions". Alternately, this residual DNA extension might reflect Mfd binding to a TRCF bubble and 542 translocating forward via continued ATP hydrolysis. Mfd recruits the repair machinery to the site of distressed polymerases during transcriptioncoupled nucleotide excision repair. During global genomic nucleotide excision repair, the damage 
the damage verification enzyme UvrB at the site (Kisker et al., 2013). Two UvrB helicases then examine the unpaired ssDNA with single-nucleotide resolution to precisely identify the damaged strand (Jaciuk et al., 2020; Pakotiprapha et al., 2012). In contrast, during transcription coupled DNA repair, Mfd recruits $\mathrm{UvrA}_{2} \mathrm{~B}$ to the site of the distressed polymerase (Ho et al., 2020). Compared to global genomic nucleotide excision repair, only a single copy of UvrB is recruited to a site of damage by Mfd through interactions with $\mathrm{VvrA}_{2}$. Thus, sequestration of the damage-containing template by Mfd, as suggested here, raises the intriguing possibility that Mfd possesses the intrinsic ability to faithfully convey the identity of the damaged strand to complete the handoff to a single UvrB via the adaptor UvrA 2 . Further elucidation of transcription-coupled DNA repair intermediates will shed light on this model. with this expectation, overexpression of Mfd was found to sensitize cells to 4-nitroquinoline, ultraviolet light and nitrofurazone (Kamarthapu et al., 2016). Based on the findings of our study, we can propose a model in which high cellular concentrations of Mfd lead to indiscriminate binding of Mfd to ECs, leading to deposition of TRCF bubble-bound translocating Mfd molecules in the wake of transcription elongation. Treatment with DNA damaging agents may serve to damage the exposed nucleobases in single-stranded DNA. Thus, the TRCF bubble may explain a hitherto mysterious property of Mfd: the ability to promote mutagenesis (Ragheb et al., 2019).

\section{Acknowledgments}

564 We thank Rachel Ann Mooney and Robert Landick (UW Madison) for generously sharing expression 565 vectors for RNA polymerase and purified NusG used in these studies. HG is supported by NIH Grant 566 1RM1GM130450 and acknowledges UOW Small Project Grant (2020/SPGA-S/04). ND, AJO, PJL, HG

567 acknowledge support by the Australian Research Council (DP210100365). AMVO acknowledges 568 support by the NIH (1RM1GM130450) and Australian Research Council (DP210100167).

\section{Author Contributions}

570 Data curation: BP, ZQ, SJ, SB, HG; Data analysis: BP, ZQ, SJ, HG, AJO, PJL; Writing-Original draft: HG; 571 Writing-review and editing: all authors; Resources: ND and AvO; Conceptualization: BP and HG; 
bioRxiv preprint doi: https://doi.org/10.1101/2021.04.02.438179; this version posted April 2, 2021. The copyright holder for this preprint (which was not certified by peer review) is the author/funder. All rights reserved. No reuse allowed without permission.

572 Supervision: HG.

\section{Declaration of Interests}

574 No competing interests.

575 


\section{Proteins used in this study}

\section{$578 \quad$ Purification of RNA polymerase}

579 RNA polymerase was purified essentially as described previously (Svetlov and Artsimovitch, 2015).

580 pIA900 was a generous gift from Irina Artsimovitch (Addgene \#104401)(Svetlov and Artsimovitch,

581 2015). plA900 was transformed into BL21( $\lambda$ DE3)recA- cells. A single colony was isolated and streaked on an ampicillin plate $\left(100 \mathrm{mg} \mathrm{L}^{-1}\right)$. Four clones were then tested for small scale protein expression (25 $\mathrm{mL}$ ) and were found to produce similar levels of overexpressed RNAP. Colony 1 was then used for overproduction. $6 \mathrm{~L}$ of culture were inoculated with an overnight culture of colony 1 and shaken for $2.5 \mathrm{~h}$ at $37{ }^{\circ} \mathrm{C}$ to reach $A_{595}=0.6$, followed by induction with a final concentration of $1 \mathrm{mM}$ IPTG. Following induction, cultures were grown for an additional $3 \mathrm{~h}$ followed by pelleting. Overproduced protein was purified from cells following the exact protocols described in (Svetlov and Artsimovitch, 2015). Following chromatographic separation over the Mono-Q column, two fractions were isolated, one corresponding to the RNAP core complex, and a second smaller fraction corresponding to the

590 holoenzyme. For this work, the RNAP core complex was isolated at a concentration of $35.7 \mathrm{mg} \mathrm{mL}^{-1}$ corresponding to $92 \mu \mathrm{M}$ in storage buffer $(50 \mathrm{mM}$ Tris/ $\mathrm{HCl} \mathrm{pH} \mathrm{7.6,} 100 \mathrm{mM} \mathrm{NaCl}, 1 \mathrm{mM}$ EDTA, $1 \mathrm{mM}$ dithiothreitol and $50 \% \mathrm{v} / \mathrm{v}$ glycerol). Purified protein was stored at $-80{ }^{\circ} \mathrm{C}$ following snap freezing with liquid $\mathrm{N}_{2}$. Aliquots in use were stored at $-20^{\circ} \mathrm{C}$.

Overproduction and purification of Mfd variants

Sequence verified $m f d$-ypet, $m f d(E 730 Q)$-ypet and $m f d(R 953 A)$-ypet genes were obtained from Aldevron. Plasmids for overexpression of $\mathrm{Mfd}, \mathrm{Mfd}(\mathrm{E} 730 \mathrm{Q})$ and $\mathrm{Mfd}(\mathrm{R} 953 \mathrm{~A})$ were created as follows: first, $m f d$ or mutant gene inserts (lacking the ypet gene) were amplified using PCR from the custom synthesized constructs using the primers (pETMCSII_Mfd_F and pETMCSII_Mfd_R; Table 1). PCR products were digested with with BamHI and EcoRI and purified over a Qiagen miniprep kit. The $m f d$

600 (or mutant) fragments were inserted between the same restriction sites in vector pSH1017 (Hamdan et al., 2002), previously isolated using electrophoretic separation and an extraction from a $1 \%(\mathrm{w} / \mathrm{v})$ 

transformed into $E$. coli DH5 $\alpha$ cells, colonies were screened for the presence of insert by colony PCR and intact $m f d$ sequences in plasmids pHG4137 (coding for wildtype Mfd), pHG42305 (Mfd(R953A)) and pHG42306 (Mfd(E730Q)) were confirmed by sequencing. $\mathrm{BL21}(\lambda \mathrm{DE} 3) \mathrm{rec} / \mathrm{pHG} 42306$ (E730Q) was grown at $37^{\circ} \mathrm{C}$ in LB medium supplemented with thymine (50 $\left.\mathrm{mg} \mathrm{L}^{-1}\right)$ and ampicillin $\left(200 \mathrm{mg} \mathrm{L}^{-1}\right)$ to $A_{600}=0.6$. To induce $\mathrm{Mfd}(\mathrm{E} 730 \mathrm{Q})$ overproduction, $1 \mathrm{mM}$ IPTG was added to the shaking culture. Cultures were grown for a further $3 \mathrm{~h}$, and then chilled in ice. Cells were harvested by centrifugation $(11,000 \times g ; 6 \mathrm{~min})$, frozen in liquid $\mathrm{N}_{2}$ and stored at $-80^{\circ} \mathrm{C}$. $\mathrm{mM}$ Tris/ $\mathrm{HCl}, \mathrm{pH}$ 7.6, $2 \mathrm{mM}$ dithiothreitol, $0.5 \mathrm{mM}$ EDTA, $20 \mathrm{mM}$ spermidine) and lysed by two passages through a French press operated at 12000 psi. Cell debris was removed from the lysate by centrifugation $(35,000 \times \mathrm{g} ; 30 \mathrm{~min})$ to yield the soluble Fraction I. Proteins in Fraction I that were then precipitated by addition of solid ammonium sulfate $\left(0.38 \mathrm{~g} \mathrm{~mL}^{-1}\right)$, followed by stirring for $30 \mathrm{~min}$, were collected by centrifugation $(38,000 \times \mathrm{g} ; 30 \mathrm{~min}$ ) and dissolved in $45 \mathrm{~mL}$ buffer $\mathrm{A}$ ( $50 \mathrm{mM}$ Tris $/ \mathrm{HCl}, \mathrm{pH}$ 7.6, $2 \mathrm{mM}$ dithiothreitol, $0.5 \mathrm{mM}$ EDTA, $10 \% \mathrm{v} / \mathrm{v}$ glycerol) containing $180 \mathrm{mM} \mathrm{NaCl}$. The solution was dialyzed against $2 \mathrm{~L}$ of the same buffer to yield Fraction II. at $1 \mathrm{~mL} \mathrm{~min} \mathrm{~m}^{-1}$ onto a column $(2.5 \times 15 \mathrm{~cm})$ of Toyopearl DEAE-650M resin that had been equilibrated

622 in buffer $\mathrm{A}+190 \mathrm{mM} \mathrm{NaCl}$. Fractions containing proteins that did not bind to the resin $(50 \mathrm{~mL}$ per sub623 fraction; $100 \mathrm{~mL}$ in total) were pooled and dialyzed against two changes of $2 \mathrm{~L}$ of buffer $\mathrm{A}$ to yield 624 Fraction III. equilibrated with buffer $A$. After the column was washed with $80 \mathrm{~mL}$ of the same buffer, $\mathrm{Mfd}(\mathrm{E} 730 \mathrm{Q})$ 
$\mathrm{Mfd}(\mathrm{E} 730 \mathrm{Q})$ eluted in a single, relatively narrow, peak centered around $80 \mathrm{mM} \mathrm{NaCl}$. Fractions containing $\mathrm{Mfd}(\mathrm{E} 730 \mathrm{Q})(40 \mathrm{~mL})$ were pooled and dialyzed against four changes of $1.2 \mathrm{~L}$ of buffer $\mathrm{B}$ (50 $\mathrm{mM} \mathrm{Na/MES,} \mathrm{pH} \mathrm{6.5,} 2$ mM dithiothreitol, 0.5 mM EDTA, 10\% v/v glycerol) to yield Fraction IV. preparations were snap frozen in liquid $\mathrm{N}_{2}$ and stored at $-80{ }^{\circ} \mathrm{C}$.

641 containing Mfd from the second DEAE (40 mL) column were pooled and directly applied at $1 \mathrm{~mL} \mathrm{~min}^{-}$

$642{ }^{1}$ onto a $2.5 \times 10 \mathrm{~cm}$ heparin Sepharose 4B that had been equilibrated with buffer $\mathrm{A}+50 \mathrm{mM} \mathrm{NaCl}$.

$643 \mathrm{Mfd}$ was eluted at $1 \mathrm{~mL} \mathrm{~min}{ }^{-1}$, in a broad peak centered around $\sim 120 \mathrm{mM} \mathrm{NaCl}$, using a linear gradient

$644(450 \mathrm{~mL})$ of $50-400 \mathrm{mM} \mathrm{NaCl}$ in buffer A. Fractions containing highly purified $\mathrm{Mfd}$ were pooled and 645 dialyzed against $2 \mathrm{~L}$ of storage buffer $(40 \mathrm{mM}$ Tris/ $\mathrm{HCl}, \mathrm{pH}$ 7.6, $100 \mathrm{mM} \mathrm{NaCl}, 4 \mathrm{mM}$ dithiothreitol, 0.5 mM EDTA, $25 \% v / v$ glycerol) to yield $115 \mathrm{mg}$ of protein at a final concentration of $40 \mu \mathrm{M}$. Purified Mfd was snap frozen in liquid $\mathrm{N}_{2}$ and aliquots were stored at $-80^{\circ} \mathrm{C}$. 
Table 1: Nucleic Acids used in this study

\begin{tabular}{|c|c|c|}
\hline Experiment & DNA & Sequence \\
\hline $\begin{array}{c}\text { pETMCSII_Mf } \\
\text { d_f }\end{array}$ & $\begin{array}{l}\text { Cloning } \\
\text { primer }\end{array}$ & $\begin{array}{l}\text { GTT TAA TCG GAT CCT AAG GAG GTT AAT TCC CGC TAT GCC } \\
\text { TGA ACA ATA TCG TTA TAC G }\end{array}$ \\
\hline $\begin{array}{c}\text { pETMCSII_Mf } \\
\text { d_R }\end{array}$ & Cloning & GGGAGCTCGAATTCTTAAGCGATCGCGTTCTCT \\
\hline $\begin{array}{c}\text { smFRET } \\
\text { (Figures 1-3) }\end{array}$ & $\begin{array}{l}\text { Non- } \\
\text { template } \\
\text { (NT70_bio) }\end{array}$ & $\begin{array}{l}\text { ATC GAG CAA CTA CTC AGA CAG CAC TAC TGC GAC TTA CAG } \\
\text { ACA TCG AGA GGG TAA TGG CGA ATA GCA CTG A /3 bioTEG/ }\end{array}$ \\
\hline $\begin{array}{c}\text { smFRET } \\
\text { (Figures 1-3) }\end{array}$ & $\begin{array}{l}\text { Template } \\
\text { (T70_31Cy3 } \\
\text { ) }\end{array}$ & $\begin{array}{l}\text { TCA GTG CTA TTC GCC ATT ACC CTC TCG ATG T/iCy3/CT GTA } \\
\text { AGT CGC AGT AGT GCT GTC TGA GTA GTT GCT CGAT }\end{array}$ \\
\hline $\begin{array}{c}\text { smFRET } \\
\text { (Figures 1-3) }\end{array}$ & $\begin{array}{l}\text { RNA(R15_4 } \\
\text { Cy5) }\end{array}$ & rArUrArU/iCy5/rArU rArUrC rGrArG rArGrG \\
\hline $\begin{array}{l}\text { smFRET } \\
\text { (Figure 4) }\end{array}$ & $\begin{array}{l}\text { bio_AS18_C } \\
\text { y5 }\end{array}$ & /5Biosg/ TGG CGA CGG CAG CGA GGC/3Cy5Sp/ \\
\hline $\begin{array}{l}\text { smFRET } \\
\text { (Figure 4) }\end{array}$ & $\begin{array}{l}\text { Cy3_dT40_ } \\
\text { S18 }\end{array}$ & $\begin{array}{l}\text { /5Cy3/TT TTT TTT TTT TTT TTT TTT TTT TTT TTT TTT TTT TTT } \\
\text { TTG CCT CGC TGC CGT CGC CA }\end{array}$ \\
\hline $\begin{array}{c}\text { SPR } \\
\text { (Supplement } \\
\text { al Figure 4) }\end{array}$ & bio_AS18 & /5Biosg/ TGG CGA CGG CAG CGA GGC \\
\hline SPR & dT40_S18 & TT TTT TTT TTT TTT TTT TTT TTT TTT TTT TTT TTT TTT TTG CCT \\
\hline
\end{tabular}




\begin{tabular}{|c|c|c|}
\hline $\begin{array}{l}\text { (Supplement } \\
\text { al Figure 4) }\end{array}$ & & CGC TGC CGT CGC CA \\
\hline $\begin{array}{c}\text { SPR } \\
\text { (Supplement } \\
\text { al Figure 4) }\end{array}$ & $\begin{array}{l}\text { bio_dT40_S } \\
18\end{array}$ & $\begin{array}{l}\text { /Biosg/TT TTT TTT TTT TTT TTT TTT TTT TTT TTT TTT TTT TTT } \\
\text { TTG CCT CGC TGC CGT CGC CA }\end{array}$ \\
\hline $\begin{array}{c}\text { SPR } \\
\text { (Supplement } \\
\text { al Figure 4) }\end{array}$ & AS18 & TGG CGA CGG CAG CGA GGC \\
\hline $\begin{array}{c}\text { SPR } \\
\text { (Supplement } \\
\text { al Figure 2) }\end{array}$ & TS_49 & $\begin{array}{l}\text { TCA GTG CTA TTC GCC ATT ACC CTC TCG ATG T/iCy3/CT GTA } \\
\text { AGT CGC AGT AGT G }\end{array}$ \\
\hline $\begin{array}{c}\text { SPR } \\
\text { (Supplement } \\
\text { al Figure 2) }\end{array}$ & NT_49_bio & $\begin{array}{l}\text { CAC TAC TGC GAC TTA CAG ACA TCG AGA GGG TAA TGG CGA } \\
\text { ATA GCA CTG A /3 bioTEG/ }\end{array}$ \\
\hline $\begin{array}{c}\text { SPR } \\
\text { (Supplement } \\
\text { al Figure 5) }\end{array}$ & $\begin{array}{l}\text { NT_49_bio } \\
\text { _bubble }\end{array}$ & $\begin{array}{l}\text { CAC TAC TGC GAC TTA CAG ACT TTT TTT TTG TAA TGG CGA ATA } \\
\text { GCA CTG A /3BioTEG/ }\end{array}$ \\
\hline $\begin{array}{c}\text { SPR } \\
\text { (Supplement } \\
\text { al Figure 2) }\end{array}$ & RNA_15 & rArUrAr UrArU rArUrC rGrArG rArGrG \\
\hline
\end{tabular}

653 All oligonucleotides were purchased from Integrated DNA Technologies (IDT, Singapore). Primed DNA

654 template for smFRET and cryo-EM was first annealed by hybridizing bio_AS18_Cy5 and Cy3_dT40_S18 
and 1.05-fold excess of the bio_AS18_Cy5 complementary strand.

\section{Single molecule FRET measurements}

SmFRET TIRF microscope setup:

659 A home built objective-type TIRF microscope based on a Nikon TiE2 model was used to record single molecule movies. FRET was measured by excitation with a $532 \mathrm{~nm}$ laser and the emissions at 555 and $647 \mathrm{~nm}$ were collected using a band-pass filter at $555 \mathrm{~nm}$ and a long-pass filter at $650 \mathrm{~nm}$. Scattered light was removed by using a $560 \mathrm{~nm}$ long pass filter. Cy3 and Cy5 signals were separated by a $638 \mathrm{~nm}$ dichroic using photometrics dual view (DV-2) and both signals were focused onto a CCD camera

664 (Hamamatsu C9 100-13), simultaneously. Data were collected at 5 frames s ${ }^{-1}$.

665 Preparation of flow cells for imaging:

666 Coverslips were prepared as described previously (Johnston et al., 2020). Quartz coverslips were cleaned with $100 \%$ ethanol and $1 \mathrm{mM} \mathrm{KOH}$. Aminosilanization of cleaned coverslips was carried out using a $1 \%(v / v)$ (3-aminopropyl)triethoxy silane (Alfa Aesar, A10668, UK) solution in acetone. PEGylation was carried out by incubating a mixture of biotinPEG-SVA MW 5000 and mPEG-SVA MW 5000 (Laysan Bio, $\mathrm{AL}$ ) at a ratio of 1:10 prepared in $50 \mathrm{mM} \mathrm{MOPS} \mathrm{pH} 7.5$ solution on the top of the silanized coverslip for 3-4 h. PEGylated coverslips were stored under dry nitrogen gas at $-20^{\circ} \mathrm{C}$. coverslip followed by a 10 min incubation. Sample flow chambers were created by sandwiching

675 polydimethylsiloxane (PDMS) on the top of the streptavidin coated coverslip. Then, blocking buffer (1

$676 \times \mathrm{TB}+0.25 \%(\mathrm{v} / \mathrm{V})$ Tween 20$)$ was injected into the channel to reduce non-specific binding of proteins

677 on the surface followed by 10-15 min incubation. This was then followed by washing with $1 \times$ TB to 678 remove unbound Tween 20.

679 In situ reconstitution of ECs for smFRET measurements: 

suspension of $200 \mu \mathrm{L}$ of $1 \times \mathrm{TB}$ and introduced into the flow cell and incubated for at least $5 \mathrm{~min}$ to promote annealing of the template DNA to the biotinylated non-template DNA resulting in formation and subsequently with $1 \times$ TB supplemented with an oxygen-scavenging system (OSS) consisting of protocatechuic acid $(2.5 \mathrm{mM})$ and protocatechuate-3,4-dioxigenase $(50 \mathrm{nM})$ to reduce photobleaching of the fluorophores and $2 \mathrm{mM}$ Trolox to reduce photo-blinking of dyes, followed by imaging. All elongation experiments in Figures 1 and 2 were performed at $100 \mu \mathrm{M}$ of each NTP or dNTP (where indicated). smFRET experiments involving NusG were performed at $500 \mathrm{nM}$ of NusG in $1 \times$ TB (Figure 2). smFRET experiments involving wildtype Mfd (Figures 3 and 4) were performed at $500 \mathrm{nM}$ of Mfd in $1 \times \mathrm{TB}$. Experiments involving $\mathrm{Mfd}(\mathrm{R} 953 \mathrm{~A})$ (Figure 3 and 4) and $\mathrm{Mfd}(\mathrm{E} 730 \mathrm{Q})$ (Figure 4) were performed at $1 \mu \mathrm{M}$ of Mfd mutant in $1 \times \mathrm{TB}$. Concentrations of ATP and ATP $\gamma \mathrm{S}$ were at $1 \mathrm{mM}$ and 0.5 $\mathrm{mM}$ respectively unless otherwise specified.

Primed DNA template (Figure 4):

Primed DNA substrate (bio_AS18_Cy5: Cy3_dT40_S18) used in smFRET experiments in Figure 4 was diluted to $50 \mathrm{pM}$ in $200 \mu \mathrm{L}$ of $1 \times \mathrm{TB}$ prior to immobilization in the flow cell.

Data Analysis

704 Single-molecule data acquisition was carried out using NIS element software. Then, single-molecule 705 intensity time trajectories and FRET-time trajectories were generated in MASH-FRET software 706 (https://rna-fretools.github.io/MASH-FRET). Further data analysis and figures are plotted using 707 custom written Python scripts. 
Surface plasmon resonance

710

Activation of SPR chip:

711

SPR experiments were performed using a BIAcore T200 instrument (GE Healthcare) using a streptavidin (SA) coated sensor chip to study the binding kinetics of Mfd to reconstituted ECs, or various DNA substrates. All experiments were carried out at $20^{\circ} \mathrm{C}$ with a flow rate of $5 \mu \mathrm{L} \mathrm{min}{ }^{-1}$, unless specified otherwise. The SA chip was activated with three sequential 1 min injections of $1 \mathrm{M} \mathrm{NaCl}, 50$ $\mathrm{mM} \mathrm{NaOH}$, then stabilized by 1 min treatment with $1 \mathrm{M} \mathrm{MgCl}_{2}$.

Immobilization of 49-mer dsDNA substrates (Supplemental Figure 2):

49-mer dsDNA substrate was prepared by hybridizing NT_49_bio (final concentration of $1 \mu \mathrm{M}$ ) with

718 two-fold excess of TS_49 in $1 \times$ TB in a final volume of $120 \mu \mathrm{L}$ by heating in a heat block at $90^{\circ} \mathrm{C}$ for 5 min followed by a slow cooling step until the room temperature was reached. NT_49_bio:TS_49 dsDNA substrate was resuspended in 1 × SPR buffer ( $30 \mathrm{mM}$ Tris/ $\mathrm{HCl}, \mathrm{pH} 7.6,40 \mathrm{mM} \mathrm{KCl}, 5 \mathrm{mM} \mathrm{MgCl}$, $0.005 \%(v / v)$ surfactant P20, $0.25 \mathrm{mM}$ EDTA, $0.5 \mathrm{mM}$ dithiothreitol) to a final concentration of $2.5 \mathrm{nM}$ (of the biotinylated oligo) and introduced onto the SPR chip for immobilization, followed by a wash step with $1 \mathrm{M} \mathrm{KCl}$. The signal from the dsDNA template corresponded to $106 \mathrm{RUs}$. Measurement of the Mfd binding affinity for the immobilized dsDNA ligand was performed as described below. Immobilization of reconstituted EC on biotinylated 49-mer dsDNA (Supplemental Figure 2): The RNAP:template (TS_49):RNA(RNA_15) complex was formed using the procedure described for in situ reconstitution of ECs for smFRET measurements. Following this, biotinylated non-template strand

728 (NT49_bio; $10 \mu \mathrm{L}$ of $10 \mu \mathrm{M}$ working stock in water) was added to the mixture and the reaction was incubated at room temperature for $15 \mathrm{~min}$. Next, $50 \mu \mathrm{L}$ of nickel resin suspension were washed and equilibrated with $1 \times$ TB. This resin resuspended in $50 \mu \mathrm{L}$ of $1 \times \mathrm{TB}$ was then added to the mixture containing the RNAP, template:RNA hybrid and non-template strand followed by incubation at $37^{\circ} \mathrm{C}$ for $15 \mathrm{~min}$ to allow binding of His-tagged RNAP complexes to the resin. The remaining steps were then performed at room temperature. First, supernatant from this suspension was isolated by 
centrifugation on a mini-benchtop centrifuge for $10 \mathrm{~s}$. The pelleted resin was washed with $150 \mu \mathrm{L}$ of $1 \times$ TB supplemented with $1 \mathrm{M} \mathrm{KCl}$ for 2 min. This suspension was then subjected to centrifugation and the supernatant was discarded. Following this, $150 \mu \mathrm{L}$ of elution buffer $(1 \times \mathrm{TB}+500 \mathrm{mM}$ imidazole) were then applied to the resin followed by gentle mixing by pipetting. Finally, the suspension was centrifuged and the eluate containing reconstituted elongation complexes was collected. All reactions containing RNAP were performed in LoBind microcentrifuge tubes (Eppendorf). The eluate was then applied to a size exclusion column (Wyatt SEC analytical column 030S5) previously equilibrated with $30 \mathrm{mM}$ Tris/ $\mathrm{HCl}, \mathrm{pH}$ 7.6, $150 \mathrm{mM} \mathrm{KCl,} \mathrm{15 \%}(\mathrm{v} / \mathrm{v})$ glycerol, $0.5 \mathrm{mM}$ EDTA, $1 \mathrm{mM}$ dithiothreitol to separate biotinylated elongation complexes from RNAP alone and collected in 200 $\mu \mathrm{L}$ fractions. Fraction 7 (F7) was found to contain the elongation complex as verified by smFRET studies. Immobilization of these reconstituted ECs was performed by resuspending $40 \mu \mathrm{L}$ of F7 in 200 $\mu \mathrm{L}$ of SPR buffer and introduction into the flow cell. Immobilization was stopped when the signal reached $1380 \mathrm{RU}$ over the background. This was followed by treatment with $1 \mathrm{M} \mathrm{KCl}\left(10 \mu \mathrm{L} \mathrm{min}{ }^{-1}\right)$ that served to displace incorrectly assembled or non-specifically bound RNAP from the chip surface. The resulting signal corresponded to $550 \mathrm{RU}$ over the background. ECs thus immobilized were found to be stable for several days. Measurement of Mfd binding affinity for the immobilized EC ligand was performed as described below.

751 Immobilization of primed DNA (Supplemental Figure 4):

Primed DNA templates were assembled in situ (on the chip surface) in two stages. First, the biotinylated strand was immobilized in three of the four channels in the chip. Single-stranded biotinylated DNA substrates (bio_dT40_S18, bio_AS18, and bio_AS18_Cy5) were diluted to $1 \mathrm{nM}$ in SPR buffer and immobilized in channels 2,3 , and 4 respectively. This corresponded to 150 RUs of bio_dT40_S18 (channel 2), 75 RUs of bio_AS18 (channel 3), and 80 RUs of bio_AS18_Cy5 (channel 4). Next, the complementary non-biotinylated strand ( $2 \mu \mathrm{M}$ in $1 \times$ SPR buffer) was introduced into the

758 flow cell to hybridize the template in situ at $5 \mu \mathrm{L} \mathrm{min}{ }^{-1}$. This led to a further increase in signal 759 corresponding to 39 RUs (channel 2, complementary strand: AS18), 138 RUs (channel 3, 
complementary strand: dT40_S18) and 115 RUs (channel 4, complementary strand: Cy3_dT40_S18) upon complete hybridization. Following this step, the flow cell was washed with $1 \mathrm{M} \mathrm{MgCl}_{2}$. Measurement of Mfd binding affinity to immobilized primed DNA template was performed as described below.

Titration of Mfd (Supplemental Figures 2 and 4):

Following the immobilization of the DNA substrates or the EC, in each case, binding studies were

Figure 2: NT_49_bio: TS_49), reconstituted EC (Supplemental Figure 2) and primed DNA substrates, measurements of Mfd binding to ssDNA substrate (bio_dT40_S18), an optimized concentration range of $[0,0.25,0.5,1,2,4,8,16] \mu \mathrm{M}$ was used. out prior to injection of Mfd. Mfd-ATP $\gamma \mathrm{S}$ was flowed in for $150 \mathrm{~s}$ in the association phase, and dissociation was observed for 200 s in 1 × SPR buffer lacking any Mfd or nucleotide cofactor.

\section{Analysis of sensorgrams:}

Sensorgrams were subtracted from the signal from unmodified flow cell (Flow channel 1), then zerosubtracted (injection at $0 \mu \mathrm{M}$ Mfd or mutant) and $R_{\text {eq }}$ values, generated by averaging response values in the sensorgram's steady-state region from the appropriate Mfd concentration range, fit against [Mfd] using a 1:1 steady state affinity (SSA) model incorporated in the BIAevaluation software 4.0.1:

$$
R_{\mathrm{eq}}=R_{\max }\left(\frac{[A]}{[A]+K_{\mathrm{D}}}\right)
$$

781 where $R_{\max }$ corresponds to the response when all the immobilized DNA ligands on the surface are saturated with the analyte $A, K_{D}$ is the dissociation constant, and $[A]$ is the concentration of analyte exported using Excel, then plotted in Origin, and final Figures prepared in Adobe Illustrator CC. 
Electron microcopy data acquisition:

$30 \mu \mathrm{L}$ of Mfd stock (40 $\mu \mathrm{M})$ were dialyzed overnight in $1 \mathrm{~L}$ of $1 \times \mathrm{TB}$ at $4{ }^{\circ} \mathrm{C}$ in a Slida-A-lyzer mini dialysis unit with a 3,000 MWCO (Thermo Fisher) to reduce the amount of glycerol in the sample. Following this, protein was recovered and concentrated to a $1.67 \mathrm{mg} \mathrm{mL}^{-1}$. For the sample with ADP$\mathrm{AlF}_{x}, \mathrm{ADP}, \mathrm{NaF}$ and $\mathrm{AlCl}_{3}$ were added to the mixture of $\mathrm{Mfd}\left(7.8 \mu \mathrm{L}\right.$ at $\left.1.6 \mathrm{mg} \mathrm{mL}^{-1}\right)$ and fluorescent oligonucleotides ( $10 \mu \mathrm{L}$ of $10 \mu \mathrm{M}$ stock) to final concentrations of 2,5 and $0.5 \mathrm{mM}$, respectively. This reaction mixture was incubated at $37^{\circ} \mathrm{C}$ for $5 \mathrm{~min}$ to allow binding of $\mathrm{Mfd}$ to the template. The samples were centrifuged at $20,000 \times \mathrm{g}$ for $10 \mathrm{~min}$ at $4{ }^{\circ} \mathrm{C}$ to clear any precipitate before grid preparation. Samples (3 $\mu \mathrm{L}$ ) were applied to glow-discharged Quantifoil R 1.2/1.3, Au 400 grids. The grids were blotted at $6{ }^{\circ} \mathrm{C}$ for $3.5 \mathrm{~s}$ at $100 \%$ humidity with no extra blot force on an FEI Vitrobot Mark IV and plunged into liquid ethane. The grids were stored in liquid nitrogen until use.

Electron microcopy data acquisition:

Data were collected using FEI Talos Arctica microscope at $200 \mathrm{kV}$ equipped with a Falcon III (FEI) $-1.9 \mu \mathrm{m}$, with a pixel size of $0.74 \AA ̊$ and a total dose of $50 \mathrm{e}^{-} \AA^{-2}$ accumulated over $50 \mathrm{~s}$ and fractionated across 50 frames.

\section{Image processing of EM micrographs} were manually selected after motion correction and CTF estimation for final processing. 110562 particles were selected after 2D classification and subjected to 3D classification in ab initio gold standard). 
810 Molecular dynamics with explicit solvent was used to fit the model of Mfd on primer DNA into the

811 cryo-EM density map (Singharoy et al., 2016). The starting model was comprised of the Mfd motor

812 domain (PDB ID 2eyq) with idealized B-DNA with the same sequence as used for the cryo-EM

813 experiments but with a dT(12) overhang instead of $d T(40)$. The system was equillibrated for $10 \mathrm{~ns}$. The

814 resulting model was then used as the basis for modelling the complex in COOT (Emsley et al., 2010)

815 with minimal distortions to protein secondary structure and DNA. The model was then subjected to

816 restrained positional refinement in phenix real_space_refine (Liebschner et al., 2019). The final model

817 is comprised of 382 residues from Mfd and 47 nucleotide residues. Model was visualized in

818 Chimera(Pettersen et al., 2004). 


\section{Figure 1: Reconstitution of a functional EC.}

823 (A) ECs were assembled on dye-labelled nucleic acids by flowing in pre-incubated RNAP-template:RNA complexes into a flow cell with immobilized biotinylated non-template strand (EC shown in grey). Following initial immobilization, a $1 \mathrm{M} \mathrm{KCl}$ wash step was introduced to remove poorly hybridized ECs from the surface. Here, only complexes containing both the Cy3-labelled template strand and Cy5-

827 labelled transcript will be observed when stably associated with the biotinylated non-template strand

828 (shown in orange). Two example FRET trajectories and temporal heat maps are provided for RNAP (B, 829 C) ( $n=318$ molecules), RNAP in the presence of dNTPs (D, E) ( $n=80$ molecules), RNAP with non830 complementary ('wrong') NTPs $(F, G)(n=179$ molecules) and the full set of NTPs $(H, I)(n=81$ 831 molecules) respectively. Fits to detected states in the FRET trajectories are shown in red. The sequence 832 of the four next correct incorporations is $G, U, A$ and $A$.

\section{Supplemental Figure 1: Walking the EC with subsets of correct rNTPs. Related to Figure 1.}

834 (A) Transition density plots for RNAP alone ( $n=318$ molecules), in the presence of dNTPs ( $n=80$ molecules), with non-complementary ('wrong') NTPs ( $n=179$ molecules) and the full set of NTPs $(n=$ 81 molecules) respectively.

837 (B) Schematic of in situ assembled FRET pair labelled ECs incubated with GTP (the next correct NTP) ( $\mathrm{n}$ $838=63$ molecules) or with GTP, ATP and UTP ( $n=87$ molecules). Temporal heat maps of the reaction for experiments conducted in the presence of GTP (C) or GTP, ATP and UTP (D) are presented here.

\section{Figure 2: Interactions between NusG and the EC}

841 (A) Schematic of FRET pair labelled ECs incubated with NusG (purple). Example FRET trajectories and

842 temporal heat maps for RNAP and NusG alone (B, C) ( $n=461$ molecules), RNAP and NusG in the 843 presence of wrong NTPs (D, E) ( $n=268$ molecules), and RNAP and NusG with the full set of NTPs (F, G) 
844 ( $n=425$ molecules). Here, NusG promotes the residence of the EC in a catalytically poised state (EC

845 shown in blue).

846 Supplemental Figure 2: Measurement of binding affinity of Mfd for dsDNA and the EC. Related to

$847 \quad$ Figures 2 and 3.

848 (A)Transition density plots for RNAP and NusG alone ( $n=461$ molecules), RNAP and NusG in the 849 presence of wrong NTPs ( $n=268$ molecules), and RNAP and NusG with the full set of NTPs ( $n=425$ 850 molecules). Here, NusG promotes the residence of the EC in a catalytically poised state (EC shown in 851 blue). Red ellipse highlights unidirectional transitions.

Schematic and SPR sensorgrams show association (150 s) and dissociation phases of serially-diluted $10-640 \mu \mathrm{M}$ Mfd-ATP $\gamma \mathrm{S}$, including a $0 \mu \mathrm{M}$ control for (B) biotinylated 49-mer dsDNA, and (C) in vitro reconstituted EC on biotinylated 49-mer DNA flown into the SPR chip. Responses at equilibrium averaged over the steady region of sensorgrams ( $\left.R_{\text {eq }}\right)$ were fit (insets) using a steady-state affinity (SSA) model to derive values of $K_{\mathrm{D}}$ (as indicated). Errors are S.E of the fit.

Figure 3: Interactions between Mfd and the EC.

858 (A) Schematic of FRET pair labelled surface immobilized ECs incubated with Mfd in solution. Two 859 example FRET trajectories and temporal heat maps are presented for RNAP and Mfd alone $(B, C)(n=$ 86075 molecules), RNAP and Mfd in the presence of $1 \mathrm{mM} \mathrm{ATP} \gamma \mathrm{S}(\mathrm{D}, \mathrm{E})(\mathrm{n}=68$ molecules), RNAP and Mfd 861 in the presence of ATP $(F, G)$ ( $n=174$ molecules), and RNAP and DNA-binding competent but translocase-deficient Mfd(R953A) in the presence of $1 \mathrm{mM} \mathrm{ATP}(\mathrm{H}, \mathrm{I})(\mathrm{n}=234$ molecules $)$.

863 Supplemental Figure 3: Interactions of ATP with the EC alone or in the presence of Mfd. Related to

\section{Figure 3.}

865 (A) Transition density plots for RNAP and Mfd alone ( $n=75$ molecules), RNAP and Mfd in the presence 866 of $1 \mathrm{mM} \mathrm{ATP} \gamma \mathrm{S}$ ( $\mathrm{m}=68$ molecules), RNAP and Mfd in the presence of ATP ( $n=174$ molecules), and 
867 RNAP and DNA-binding competent but translocase-deficient Mfd(R953A) in the presence of $1 \mathrm{mM}$ ATP

868 ( $n=234$ molecules). Red ellipses highlight irreversible transitions.

869 (B) Temporal heat map (upper panel) and transition density plot (lower panel) of FRET pair labelled 870 RNAP EC in the presence of $0.5 \mathrm{mM}$ ATP $\gamma \mathrm{S}$ alone $(\mathrm{n}=124$ molecules).

871 (C) Distribution of the catalytically-poised mid-FRET state for the indicated concentrations of ATP and

872 (D) Dependence of the lifetime $\left(\tau_{\text {off }}\right)$ of mid-FRET state for Mfd-RNAP ECs as a function of ATP 873 concentration in the range from $10 \mu \mathrm{M}$ to $1 \mathrm{mM}$ ATP $(n=43,24,77,32$ molecules respectively). Plots 874 represent dwell-time distributions of the surviving fraction of the population in the mid-FRET state fit 875 to a single exponential decay (red curve) to the data. Errors represent S.E to the fit.

876 (E) Temporal heat maps of FRET pair labelled RNAP EC when titrated with ATP in the range of $0.1 \mu \mathrm{M}$ 877 to $1 \mathrm{mM}(\mathrm{n}=151,82,81,76,157$ molecules respectively) as indicated.

\section{Figure 4: Interactions between Mfd and a primed-DNA template}

879 (A) Schematic of FRET pair labelled primed DNA template comprised of an 18-mer dsDNA and a 5' 40mer poly-dT overhang to assess interactions of Mfd with ssDNA. Two example FRET trajectories, temporal heat maps and transition density plots for primed DNA alone $(B, C)(n=100$ molecules), Mfd in the presence of ATP $\gamma S(D, E)$ ( $n=94$ molecules), Mfd in the presence of ATP $(F, G)(n=203$ molecules), and $\operatorname{Mfd}(\mathrm{R} 953 \mathrm{~A})$ in the presence of $\operatorname{ATP}(\mathrm{H}, \mathrm{I})(\mathrm{n}=105$ molecules $)$ are presented here. 
890 Schematic and SPR sensorgrams show association (150 s) and dissociation phases of serially-diluted

$891 \quad 10-640 \mu \mathrm{M}$ Mfd-ATP $\gamma \mathrm{S}$, including a $0 \mu \mathrm{M}$ control for (B) primed DNA substrate (biotinylated 18-mer

892 dsDNA with $\mathrm{dT}_{40}$ overhang) (C) “flipped” primed DNA substrate (18-mer dsDNA with biotinylated $\mathrm{dT}_{40}$

893 overhang) (D) FRET pair labelled primed DNA substrate (biotinylated 18-mer dsDNA with dT 40

894 overhang) and (E) 58-mer ssDNA containing the $\mathrm{dT}_{40}$ sequence. Responses at equilibrium averaged

895 over the steady region of sensorgrams $\left(R_{\text {eq }}\right)$ were fit (insets) using a steady-state affinity (SSA) model

896 to derive values of $K_{\mathrm{D}}$ (as indicated). Errors are S.E of the fit.

897 (F) Temporal heat map and (G) transition density plot for ATPase mutant Mfd(E730Q) binding to FRET

898 pair labelled primed DNA template used in Figure $4(n=103$ molecules).

899 (H) For convenience, kernel density estimations of the ensemble FRET distributions read as projections

900 along the ordinate in the heat-maps presented in Figure 4 (panels C, E, G, I) are presented to enable 901 direct visual comparisons.

\section{Figure 5: Interactions between Mfd and primed DNA}

903 (A) Views of the cryo-EM reconstruction of Mfd bound to the FRET pair labelled DNA substrate (used 904 in Figure 4).

905 (B) Front view of the molecular model derived from the cryo-EM reconstruction showing domain 5 906 (tan), domain6 (olive), template (dark red) and non-template (pink) DNA. Mfd (residues 575-956) and 907 DNA (template: residues 28-58; non-template: 1-18) are shown in surface representation to illustrate 908 the path of the DNA.

909 (C) Top view of molecular model showing TRG motif straddling the DNA. The unpaired 5' poly dT 910 overhang is colored as follows: dT1-dT3 (pink), dT4-dT6 (salmon), dT7-dT9 (sandy brown) and 911 dT11-dT12 (brown). R953 and R929 are positioned to contact the ssDNA overhang at 3 and 6 residues away from the junction respectively. 
913 (D) Model suggests dsDNA near the junction is melted to form the TRCF bubble. (F) Path of the DNA is

914 lined by highly conserved residues in Mfd across bacteria: basic residues (Lys, Arg, His) are shown in

915 blue, polar residues (Thr, Asn) are shown in gold, and aromatic residues (Phe, Tyr) are shown in green.

916 Domain 5 corresponds to residues 549 - 780, and domain 6 corresponds to residues 781-990 in Mfd.

917 Labels are provided in (G).

\section{Supplemental Figure 5: EM reconstruction of Mfd. Related to Figure 5.}

919 (A) Overview of image processing using CryoSPARC. 110562 particles were selected after 2D 920 classification for ab initio reconstruction and heterogeneous refinement leading to four classes of 3D 921 reconstructions. Of these, 31718 particles that yielded the most complete reconstruction were 922 subjected to homogenous refinement to generate the map presented here. The colormap applied to 923 the reconstruction represents local resolution. (B) Gold-standard Fourier shell correlation curves of 924 the reconstruction reported a resolution of $5.2 \AA$ (C) angular distribution of particle projections (D) 925 Measurement of dissociation constant of Mfd for a 49-mer dsDNA template containing a 9 nucleotide non-complementary bubble where the complementary residues were replaced by $\mathrm{dT}_{9}$. Errors reflect S.E of the fit. Figure 6: Model for Mfd-mediated transcription modulation binding and hydrolysis are required for stable association of Mfd with the EC. Following, a brief period of ATP-hydrolysis dependent translocation of Mfd on upstream dsDNA, Mfd loads on to the EC. This results in chaperoning of the EC from catalytically inactive state(s) into a catalytically poised molecular

933 timer intermediate (D) with a lifetime of $\sim 6 \mathrm{~s}$. In this ATP-bound state, we propose that Mfd melts the 934 upstream DNA to form an extended transcription-repair coupling factor - transcription ("TRCF-T") 935 bubble. In this state R953 contacts the template ssDNA in the TRCF-T bubble. Melting of this upstream 
937 NTPs on an undamaged template, transcription can be resumed. (F) On damaged templates or under

938 conditions of nucleotide starvation or in the presence of impediments to the progress of the EC, the

939 bound ATP is hydrolyzed to promote transcription termination. In our experiments this is seen as the

940 loss of RNA. We propose that in either case continued translocation occurs in a manner in which the

941 ATPase activity of Mfd translocates the TRCF bubble forward until Mfd interacts with its interacting

942 partners in the repair machinery.

943 
945

946

947

948

949

950

951

952

953

954

955

956

957

958

959

960

961

962

963

964

965

966

967

968

969

970

971

972

973

974

975

976

977

978

979

980

981

982

983

984

985

986

987

988

Abdelkareem, M., Saint-Andre, C., Takacs, M., Papai, G., Crucifix, C., Guo, X., Ortiz, J., and Weixlbaumer, A. (2019). Structural Basis of Transcription: RNA Polymerase Backtracking and Its Reactivation. Mol Cell 75, 298-309 e294.

Andrecka, J., Lewis, R., Bruckner, F., Lehmann, E., Cramer, P., and Michaelis, J. (2008). Singlemolecule tracking of mRNA exiting from RNA polymerase II. Proc Natl Acad Sci U S A 105, 135140.

Belogurov, G.A., and Artsimovitch, I. (2019). The Mechanisms of Substrate Selection, Catalysis, and Translocation by the Elongating RNA Polymerase. J Mol Biol 431, 3975-4006. Borukhov, S., Sagitov, V., and Goldfarb, A. (1993). Transcript cleavage factors from E. coli. Cell 72, 459-466.

Bronson, J.E., Fei, J., Hofman, J.M., Gonzalez, R.L., Jr., and Wiggins, C.H. (2009). Learning Rates and States from Biophysical Time Series: A Bayesian Approach to Model Selection and SingleMolecule FRET Data. Biophys J 97, 3196-3205.

Brugger, C., Zhang, C., Suhanovsky, M.M., Kim, D.D., Sinclair, A.N., Lyumkis, D., and Deaconescu, A.M. (2020). Molecular determinants for dsDNA translocation by the transcription-repair coupling and evolvability factor Mfd. Nat Commun 11, 3740.

Burova, E., Hung, S.C., Sagitov, V., Stitt, B.L., and Gottesman, M.E. (1995). Escherichia coli NusG Protein Stimulates Transcription Elongation Rates In Vivo and In Vitro. Journal of bacteriology 177, 1388-1392.

Byrd, A.K., and Raney, K.D. (2012). Superfamily 2 helicases. Front Biosci (Landmark Ed) 17, 2070-2088.

Chambers, A.L., Smith, A.J., and Savery, N.J. (2003). A DNA translocation motif in the bacterial transcription-repair coupling factor, Mfd. Nucleic Acids Res 31, 6409-6418.

Deaconescu, A.M., Chambers, A.L., Smith, A.J., Nickels, B.E., Hochschild, A., Savery, N.J., and Darst, S.A. (2006). Structural Basis for Bacterial Transcription-Coupled DNA Repair. Cell 124, 507-520.

Deaconescu, A.M., Sevostyanova, A., Artsimovitch, I., and Grigorieff, N. (2012). Nucleotide excision repair (NER) machinery recruitment by the transcription-repair coupling factor involves unmasking of a conserved intramolecular interface. Proc Natl Acad Sci U S A 109, 3353-3358.

Duan, M., Selvam, K., Wyrick, J.J., and Mao, P. (2020). Genome-wide role of Rad26 in promoting transcription-coupled nucleotide excision repair in yeast chromatin. Proc Natl Acad Sci U S A 117, 18608-18616.

Emsley, P., Lohkamp, B., Scott, W.G., and Cowtan, K. (2010). Features and development of Coot. Acta Crystallogr D Biol Crystallogr 66, 486-501.

Fan, J., Leroux-Coyau, M., Savery, N.J., and Strick, T.R. (2016). Reconstruction of bacterial transcription-coupled repair at single-molecule resolution. Nature 536, 234-237.

Gabizon, R., Lee, A., Vahedian-Movahed, H., Ebright, R.H., and Bustamante, C.J. (2018). Pause sequences facilitate entry into long-lived paused states by reducing RNA polymerase transcription rates. Nat Commun 9, 2930.

Ghodke, H., Ho, H.N., and van Oijen, A.M. (2020). Single-molecule live-cell imaging visualizes parallel pathways of prokaryotic nucleotide excision repair. Nat Commun 11, 1477.

Graves, E.T., Duboc, C., Fan, J., Stransky, F., Leroux-Coyau, M., and Strick, T.R. (2015). A dynamic DNA-repair complex observed by correlative single-molecule nanomanipulation and 
fluorescence. Nat Struct Mol Biol 22, 452-457.

Haines, N.M., Kim, Y.I., Smith, A.J., and Savery, N.J. (2014). Stalled transcription complexes promote DNA repair at a distance. Proc Natl Acad Sci U S A 111, 4037-4042.

Hamdan, S., Bulloch, E.M., Thompson, P.R., Beck, J.L., Yang, J.Y., Crowther, J.A., Lilley, P.E., Carr, P.D., Ollis, D.L., Brown, S.E., et al. (2002). Hydrolysis of the 5'-p-nitrophenyl ester of TMP by the proofreading exonuclease (epsilon) subunit of Escherichia coli DNA polymerase III. Biochemistry 41, 5266-5275.

Herbert, K.M., Zhou, J., Mooney, R.A., Porta, A.L., Landick, R., and Block, S.M. (2010). E. coli NusG Inhibits Backtracking and Accelerates Pause-Free Transcription by Promoting Forward Translocation of RNA Polymerase. J Mol Biol 399, 17-30.

Ho, H.N., van Oijen, A.M., and Ghodke, H. (2018). The transcription-repair coupling factor Mfd associates with RNA polymerase in the absence of exogenous damage. Nat Commun 9, 1570. Ho, H.N., van Oijen, A.M., and Ghodke, H. (2020). Single-molecule imaging reveals molecular coupling between transcription and DNA repair machinery in live cells. Nat Commun 11, 1478. Ho Wan, K., Smith, A.J., Westblade, L.F., Joly, N., Grange, W., Zorman, S., Darst, S.A., Savery, N.J., and Strick, T.R. (2012). Initiation of transcription-coupled repair characterized at singlemolecule resolution. Nature 490, 431-434.

Hopfield, J.J. (1974). Kinetic proofreading: a new mechanism for reducing errors in biosynthetic processes requiring high specificity. Proc Natl Acad Sci U S A 71, 4135-4139.

Jaciuk, M., Swuec, P., Gaur, V., Kasprzak, J.M., Renault, L., Dobrychlop, M., Nirwal, S., Bujnicki, J.M., Costa, A., and Nowotny, M. (2020). A combined structural and biochemical approach reveals translocation and stalling of UvrB on the DNA lesion as a mechanism of damage verification in bacterial nucleotide excision repair. DNA Repair (Amst) 85, 102746.

Johnston, C.L., Marzano, N.R., Paudel, B.P., Wright, G., Benesch, J.L.P., van Oijen, A.M., and Ecroyd, H. (2020). Single-molecule fluorescence-based approach reveals novel mechanistic insights into human small heat shock protein chaperone function. J Biol Chem.

Kamarthapu, V., Epshtein, V., Benjamin, B., Proshkin, S., Mironov, A., Cashel, M., and Nudler, E. (2016). ppGpp couples transcription to DNA repair in E. coli. Science 352, 993-996.

Kang, J.Y., Llewellyn, E., Chen, J., Olinares, P.D.B., Brewer, J., Chait, B.T., Campbell, E.A., and Darst, S.A. (2021). Structural basis for transcription complex disruption by the Mfd translocase. Elife 10.

Kang, J.Y., Mooney, R.A., Nedialkov, Y., Saba, J., Mishanina, T.V., Artsimovitch, I., Landick, R., and Darst, S.A. (2018). Structural Basis for Transcript Elongation Control by NusG Family Universal Regulators. Cell 173, 1650-1662 e1614.

Kisker, C., Kuper, J., and Van Houten, B. (2013). Prokaryotic Nucleotide Excision Repair. Cold Spring Harb Perspect Biol 5, a012591.

Komissarova, N., and Kashlev, M. (1997). Transcriptional arrest: Escherichia coli RNA polymerase translocates backward, leaving the $3^{\prime}$ end of the RNA intact and extruded. Proc Natl Acad Sci U S A 94, 1755-1760.

Komissarova, N., Kireeva, M.L., Becker, J., Sidorenkov, I., and Kashlev, M. (2003). Engineering of elongation complexes of bacterial and yeast RNA polymerases. Methods Enzymol 371, 233251.

Le, T.T., Yang, Y., Tan, C., Suhanovsky, M.M., Fulbright, R.M., Jr., Inman, J.T., Li, M., Lee, J., Perelman, S., Roberts, J.W., et al. (2017). Mfd Dynamically Regulates Transcription via a Release and Catch-Up Mechanism. Cell.

Liebschner, D., Afonine, P.V., Baker, M.L., Bunkoczi, G., Chen, V.B., Croll, T.I., Hintze, B., Hung, L.W., Jain, S., McCoy, A.J., et al. (2019). Macromolecular structure determination using X-rays, 
1036

1037

1038

1039

1040

1041

1042

1043

1044

1045

1046

1047

1048

1049

1050

1051

1052

1053

1054

1055

1056

1057

1058

1059

1060

1061

1062

1063

1064

1065

1066

1067

1068

1069

1070

1071

1072

1073

1074

1075

1076

1077

1078

1079

1080

1081

1082 neutrons and electrons: recent developments in Phenix. Acta Crystallogr D Struct Biol 75, 861877.

Mahdi, A.A., Briggs, G.S., Sharples, G.J., Wen, Q., and Lloyd, R.G. (2003). A model for dsDNA translocation revealed by a structural motif common to RecG and Mfd proteins. EMBO J 22, 724-734.

Manelyte, L., Kim, Y.I., Smith, A.J., Smith, R.M., and Savery, N.J. (2010). Regulation and Rate Enhancement during Transcription-Coupled DNA Repair. Mol Cell 40, 714-724.

Mayne, L.V., and Lehmann, A.R. (1982). Failure of RNA synthesis to recover after UV irradiation: an early defect in cells from individuals with Cockayne's syndrome and xeroderma pigmentosum. Cancer Res 42, 1473-1478.

Mellon, I., and Hanawalt, P.C. (1989). Induction of the Escherichia coli lactose operon selectively increases repair of its transcribed DNA strand. Nature 342, 95-98.

Muftuoglu, M., Sharma, S., Thorslund, T., Stevnsner, T., Soerensen, M.M., Brosh, R.M., Jr., and Bohr, V.A. (2006). Cockayne syndrome group B protein has novel strand annealing and exchange activities. Nucleic Acids Res 34, 295-304.

Pakotiprapha, D., Samuels, M., Shen, K., Hu, J.H., and Jeruzalmi, D. (2012). Structure and mechanism of the UvrA-UvrB DNA damage sensor. Nat Struct Mol Biol 19, 291-298.

Park, J., Myong, S., Niedziela-Majka, A., Lee, K.S., Yu, J., Lohman, T.M., and Ha, T. (2010). PcrA helicase dismantles RecA filaments by reeling in DNA in uniform steps. Cell 142, 544-555.

Park, J.S., Marr, M.T., and Roberts, J.W. (2002). E. coli Transcription Repair Coupling Factor (Mfd protein) Rescues Arrested Complexes by Promoting Forward Translocation. Cell 109, 757-767.

Pettersen, E.F., Goddard, T.D., Huang, C.C., Couch, G.S., Greenblatt, D.M., Meng, E.C., and Ferrin, T.E. (2004). UCSF Chimera--a visualization system for exploratory research and analysis. J Comput Chem 25, 1605-1612.

Proshkin, S.A., and Mironov, A.S. (2016). Stalled RNA Polymerase Is a Target of the Mfd Factor. Mol Biol (Mosk) 50, 381-384.

Punjani, A., Rubinstein, J.L., Fleet, D.J., and Brubaker, M.A. (2017). cryoSPARC: algorithms for rapid unsupervised cryo-EM structure determination. Nat Methods 14, 290-296.

Ragheb, M.N., Merrikh, C., Browning, K., and Merrikh, H. (2021). Mfd regulates RNA polymerase association with hard-to-transcribe regions in vivo, especially those with structured RNAs. Proc Natl Acad Sci U S A 118.

Ragheb, M.N., Thomason, M.K., Hsu, C., Nugent, P., Gage, J., Samadpour, A.N., Kariisa, A., Merrikh, C.N., Miller, S.I., Sherman, D.R., et al. (2019). Inhibiting the Evolution of Antibiotic Resistance. Mol Cell 73, 157-165 e155.

Selby, C.P., and Sancar, A. (1993a). Molecular Mechanism of Transcription-Repair Coupling. Science 260, 53-58.

Selby, C.P., and Sancar, A. (1993b). Transcription-Repair Coupling and Mutation Frequency Decline. Journal of bacteriology 175, 7509-7514.

Selby, C.P., and Sancar, A. (1995). Structure and Function of Transcription-Repair Coupling Factor. I. Structural Domains and Binding Properties. J Biol Chem 270, 4882-4889.

Selby, C.P., and Sancar, A. (1997). Cockayne syndrome group B protein enhances elongation by RNA polymerase II. Proc Natl Acad Sci U S A 94, 11205-11209.

Shi, J., Wen, A., Zhao, M., Jin, S., You, L., Shi, Y., Dong, S., Hua, X., Zhang, Y., and Feng, Y. (2020). Structural basis of Mfd-dependent transcription termination. Nucleic Acids Res.

Singharoy, A., Teo, I., McGreevy, R., Stone, J.E., Zhao, J., and Schulten, K. (2016). Molecular dynamics-based refinement and validation for sub-5 A cryo-electron microscopy maps. elife 
5.

Smith, A.J., Pernstich, C., and Savery, N.J. (2012). Multipartite control of the DNA translocase, Mfd. Nucleic Acids Res 40, 10408-10416.

Smith, A.J., Szczelkun, M.D., and Savery, N.J. (2007). Controlling the motor activity of a transcription-repair coupling factor: autoinhibition and the role of RNA polymerase. Nucleic Acids Res 35, 1802-1811.

Svetlov, V., and Artsimovitch, I. (2015). Purification of bacterial RNA polymerase: tools and protocols. Methods Mol Biol 1276, 13-29.

1091 Svetlov, V., Vassylyev, D.G., and Artsimovitch, I. (2004). Discrimination against Deoxyribonucleotide Substrates by Bacterial RNA Polymerase. J Biol Chem 279, 38087-38090. Troelstra, C., van Gool, A., de Wit, J., Vermeulen, W., Bootsma, D., and Hoeijmakers, J.H. (1992). ERCC6, a member of a subfamily of putative helicases, is involved in Cockayne's syndrome and preferential repair of active genes. Cell 71, 939-953. van der Weegen, Y., Golan-Berman, H., Mevissen, T.E.T., Apelt, K., Gonzalez-Prieto, R., Goedhart, J., Heilbrun, E.E., Vertegaal, A.C.O., van den Heuvel, D., Walter, J.C., et al. (2020). The cooperative action of CSB, CSA, and UVSSA target TFIIH to DNA damage-stalled RNA polymerase II. Nat Commun 11, 2104.

1100 Venema, J., Mullenders, L.H., Natarajan, A.T., van Zeeland, A.A., and Mayne, L.V. (1990). The genetic defect in Cockayne syndrome is associated with a defect in repair of UV-induced DNA damage in transcriptionally active DNA. Proc Natl Acad Sci U S A 87, 4707-4711. Wang, B., and Artsimovitch, I. (2020). NusG, an Ancient Yet Rapidly Evolving Transcription 1104 Factor. Front Microbiol 11, 619618.

1105 Xu, J., Lahiri, I., Wang, W., Wier, A., Cianfrocco, M.A., Chong, J., Hare, A.A., Dervan, P.B., DiMaio, F., Leschziner, A.E., et al. (2017). Structural basis for the initiation of eukaryotic transcription-coupled DNA repair. Nature 551, 653-657. 


\section{Supplemental Information}

1111

1112 Mechanism of transcription modulation by the transcription-repair coupling factor

1113 Bishnu Paudel ${ }^{1,2}$, Zhi-Qiang $\mathrm{Xu}^{1,2}$, Slobodan Jergic ${ }^{1,2}$, Aaron J Oakley ${ }^{1,2}$, Nischal Sharma ${ }^{1,2}$,

1114 Simon HJ Brown ${ }^{1,2}$, James C Bouwer ${ }^{1,2}$, Peter J Lewis ${ }^{3}$, Nicholas E Dixon ${ }^{1,2}$, Antoine M van

1115 Oijen $^{1,2}$, Harshad Ghodke ${ }^{1,2 *}$

$1116{ }^{1}$ Molecular Horizons and School of Chemistry and Molecular Bioscience, University of Wollongong,

1117 Wollongong, Australia

11182 Illawarra Health and Medical Research Institute, Wollongong, Australia

$1119{ }^{3}$ School of Environmental and Life Sciences, University of Newcastle, Callaghan, NSW 2308, Australia

1120 Correspondence:

1121 Harshad Ghodke, Molecular Horizons and School of Chemistry and Molecular Bioscience, University

1122 of Wollongong, Wollongong, New South Wales, 2522, Australia

1123 


\section{Supplemental Note 1}

1125 SPR measurements of the strength of interaction between Mfd and this DNA substrate in the presence

1126 of ATP $\gamma$ S revealed a dissociation constant that is somewhat higher $\left(K_{\mathrm{D}}=300 \pm 10 \mathrm{nM}\right)$ than that of

1127 dsDNA (170 $\pm 20 \mathrm{nM}$; Supplemental Figure 4B-D compared to Supplemental Figure 2B). To monitor

1128 changes in the distance between the junction and the $5^{\prime}$ end, we introduced a FRET pair on either end

1129 of the ssDNA overhang (Figure 4A). SPR measurements revealed a binding affinity of $390 \pm 10 \mathrm{nM}$ for

1130 the binding of Mfd to the FRET pair labelled substrate (Supplemental Figure 4D). 
Figure 1

A

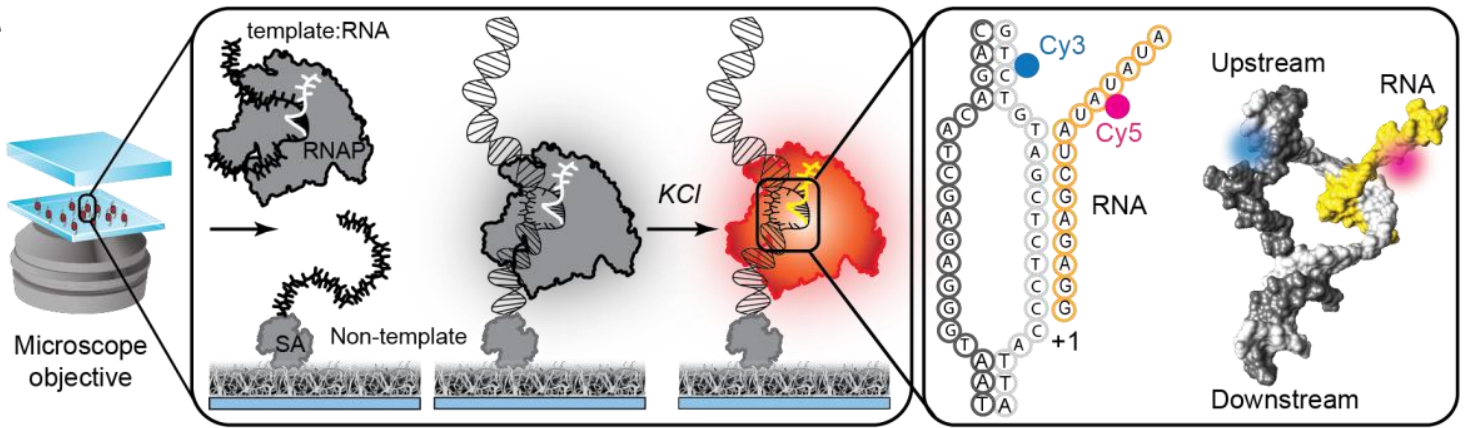

B
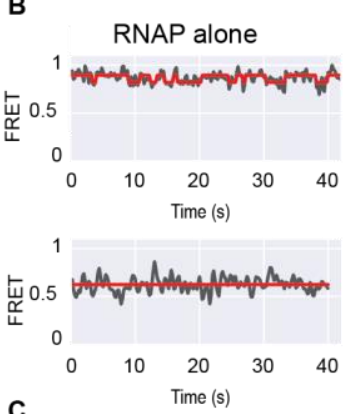

C

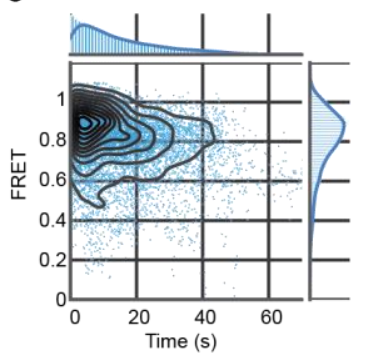

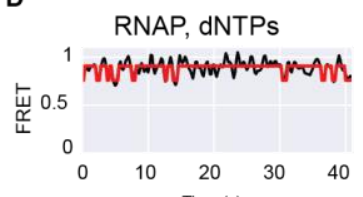

$\mathbf{F}$
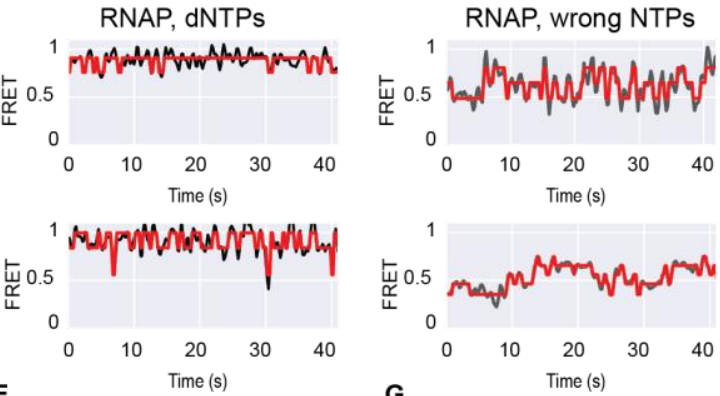

E

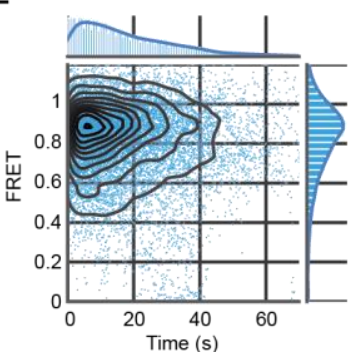

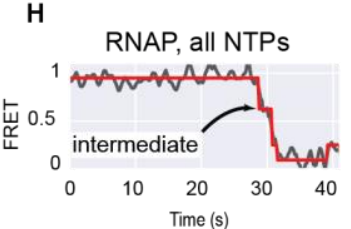
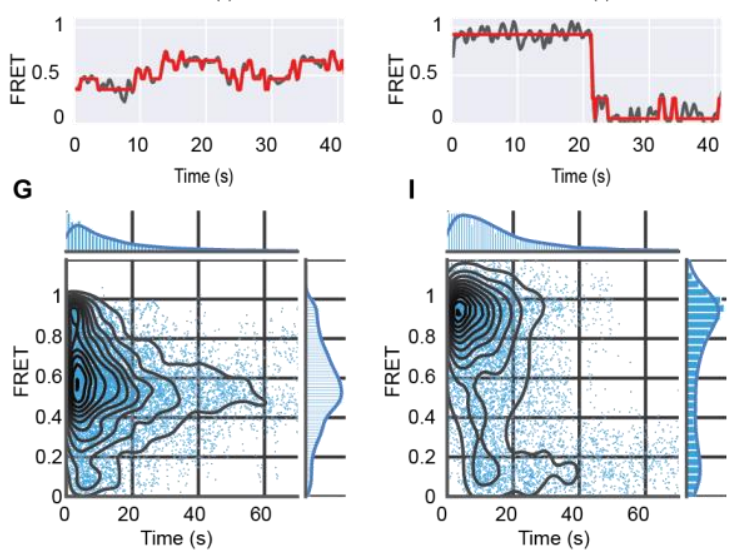

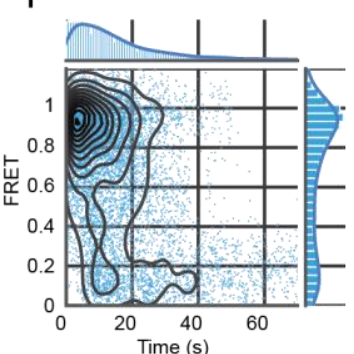


bioRxiv preprint doi: https://doi.org/10.1101/2021.04.02.438179; this version posted April 2, 2021. The copyright holder for this preprint (which was not certified by peer review) is the author/funder. All rights reserved. No reuse allowed without permission.

\section{Supplemental Figure 1}
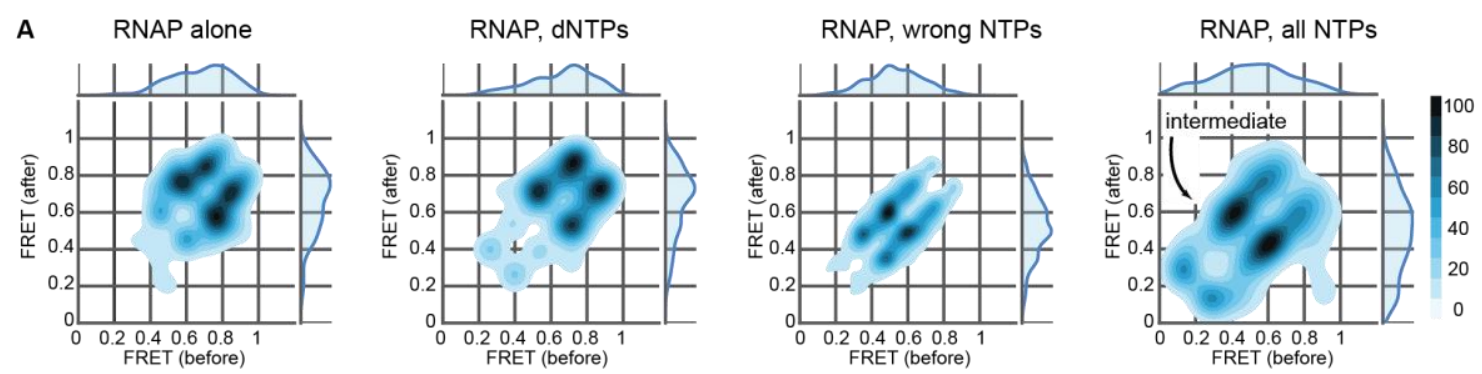

B

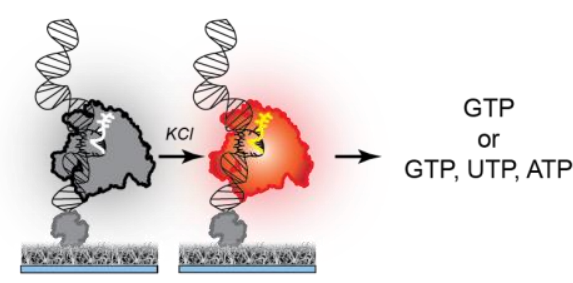

GTP

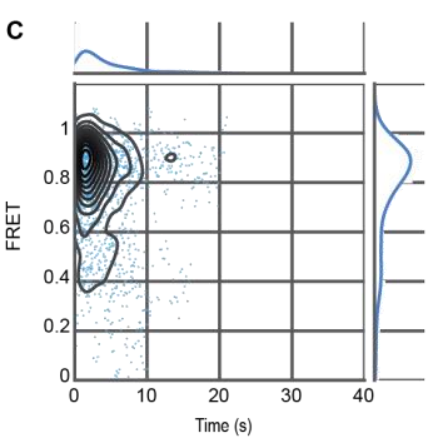

GTP, UTP, ATP

D

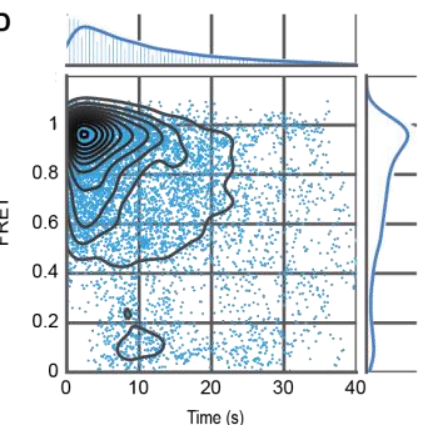


Figure 2

A
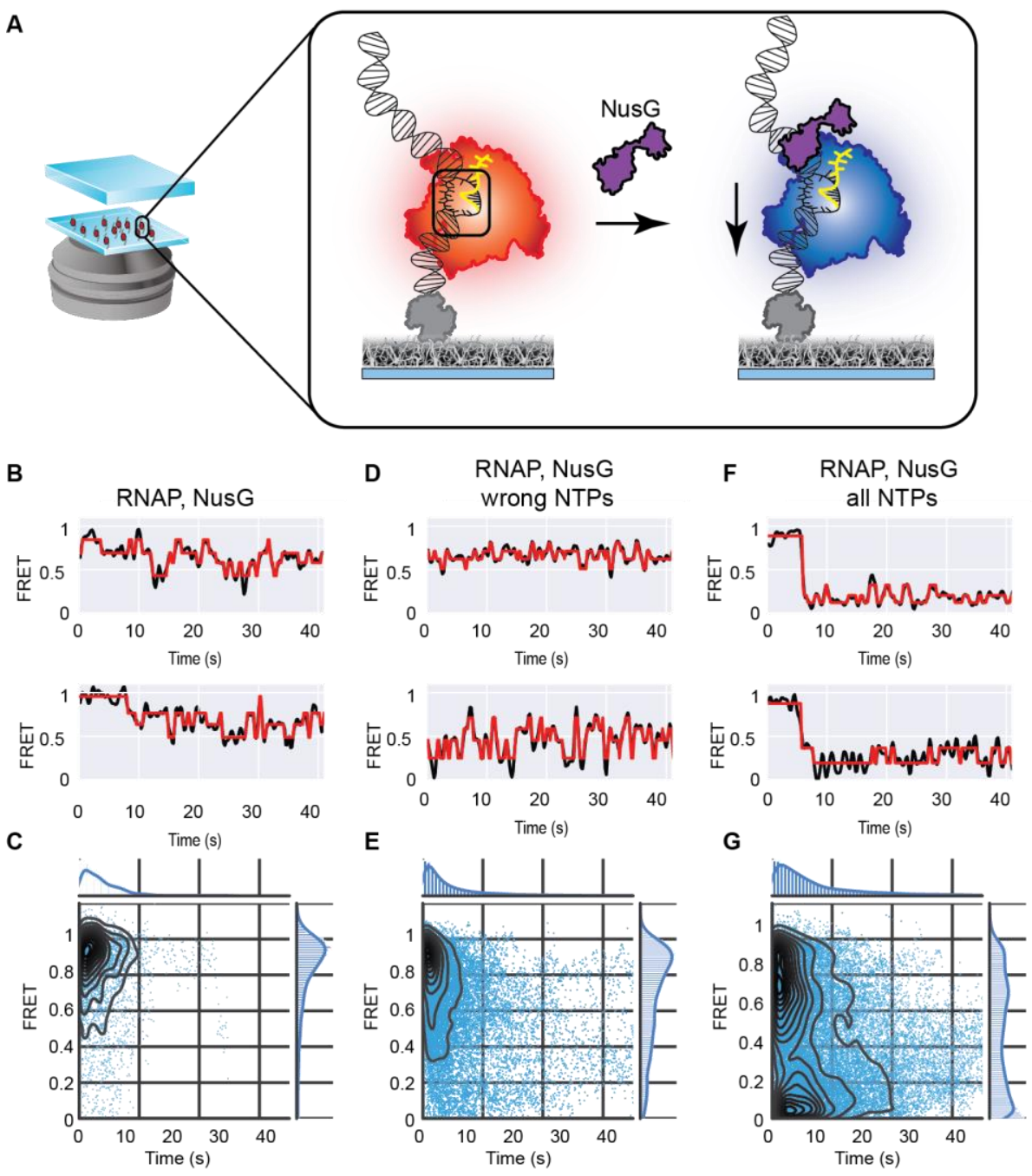
bioRxiv preprint doi: https://doi.org/10.1101/2021.04.02.438179; this version posted April 2, 2021. The copyright holder for this preprint (which was not certified by peer review) is the author/funder. All rights reserved. No reuse allowed without permission.

\section{Supplemental Figure 2}

A

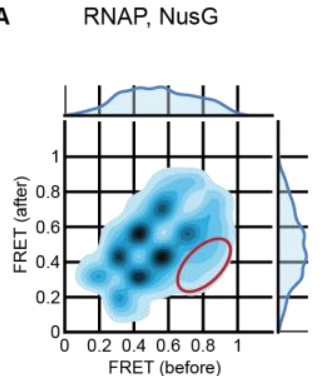

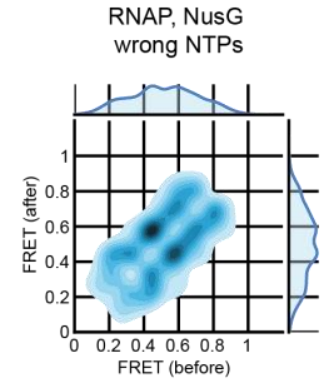

RNAP, NusG

all NTPs

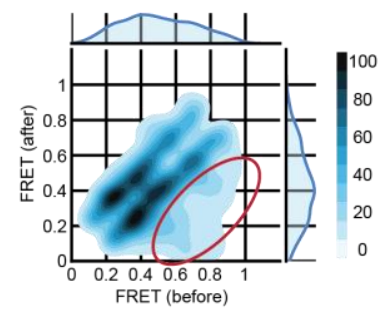

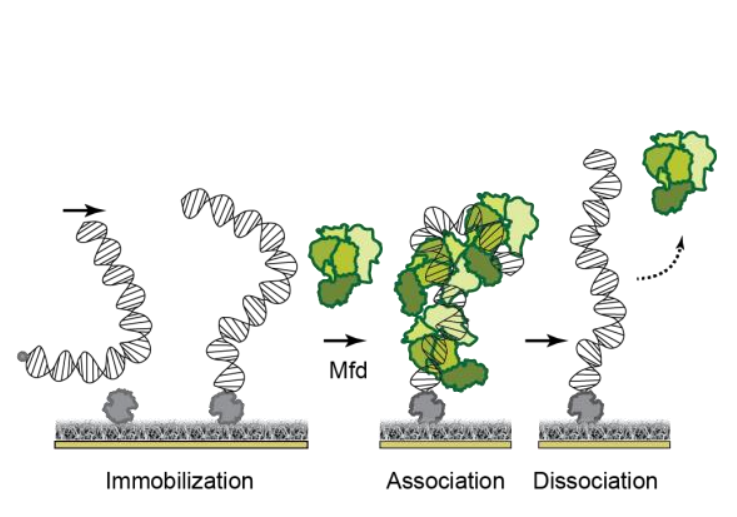

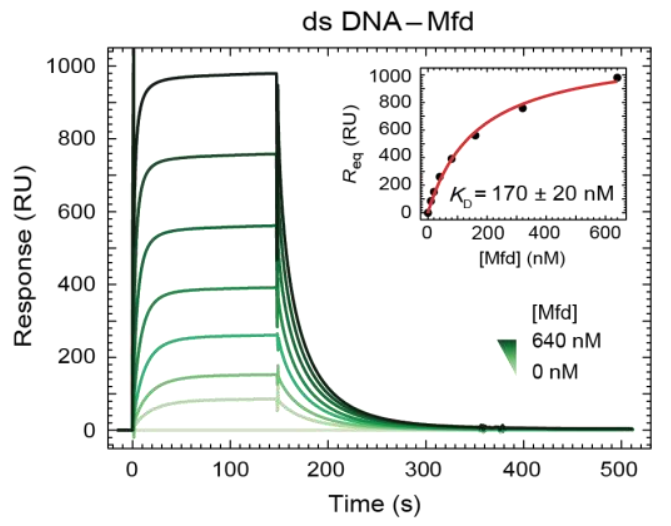

C

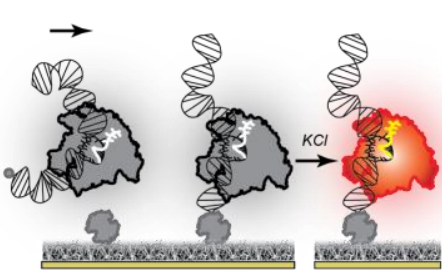

Immobilization

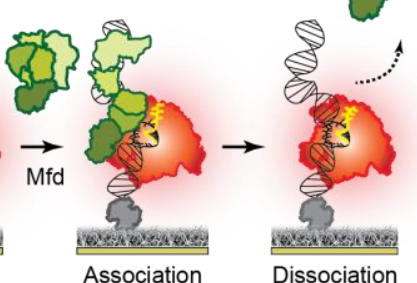

Association

Dissociation

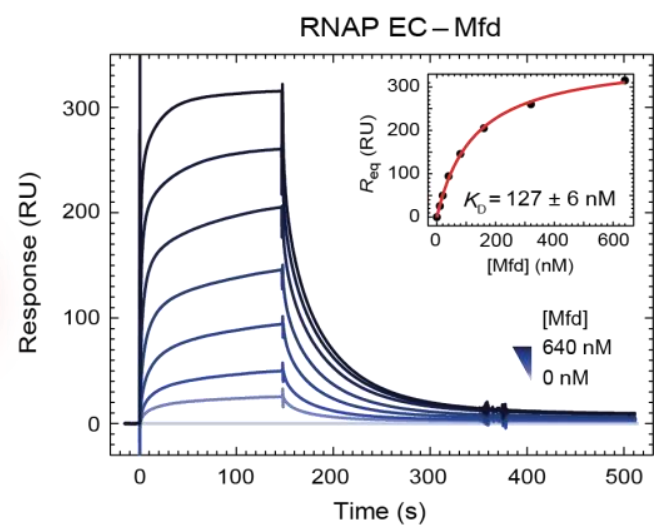


bioRxiv preprint doi: https://doi.org/10.1101/2021.04.02.438179; this version posted April 2, 2021. The copyright holder for this preprint (which was not certified by peer review) is the author/funder. All rights reserved. No reuse allowed without permission.

Figure 3

A

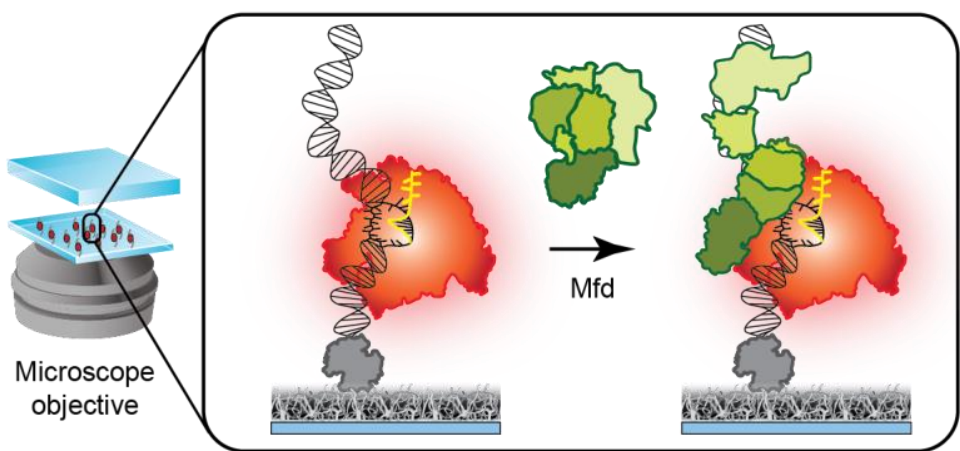

B

D

F

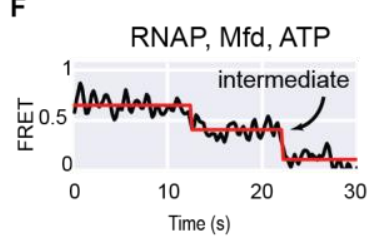

RNAP
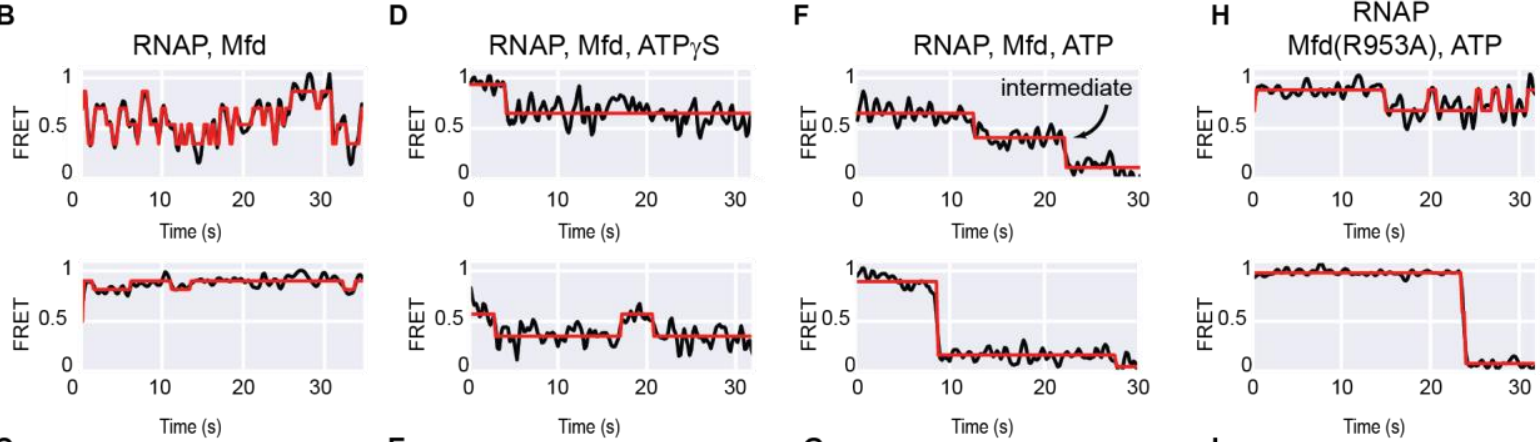

C

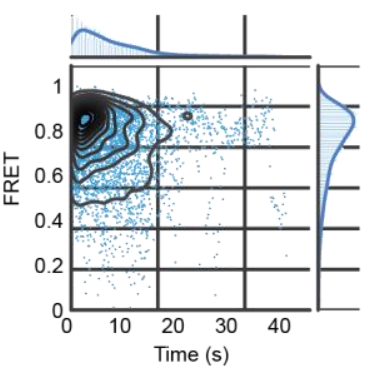

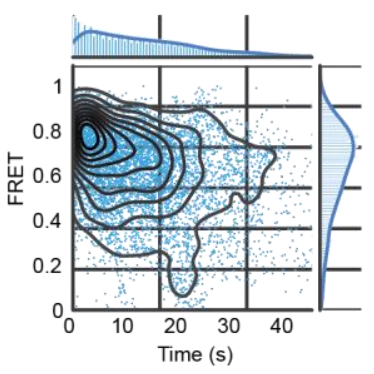

G

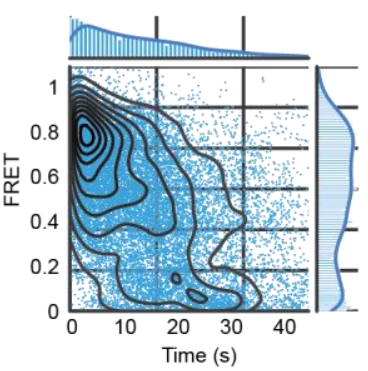

I

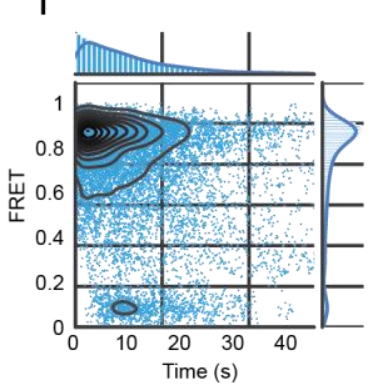


bioRxiv preprint doi: https://doi.org/10.1101/2021.04.02.438179; this version posted April 2, 2021. The copyright holder for this preprint (which was not certified by peer review) is the author/funder. All rights reserved. No reuse allowed without permission.

\section{Supplemental Figure 3}

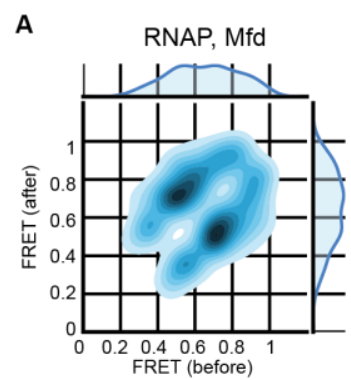

RNAP, Mfd, ATP

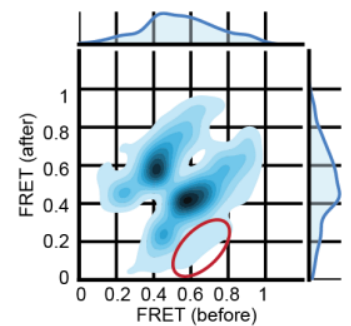

C

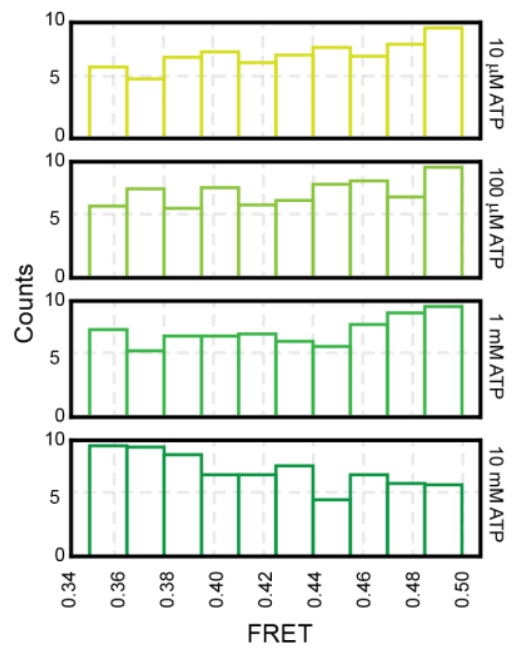

RNAP, Mfd, ATP $\gamma \mathrm{S}$

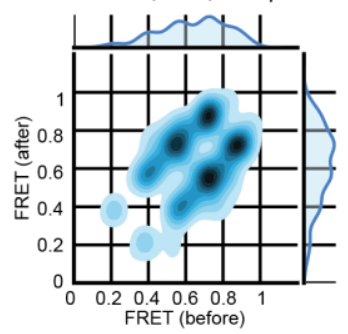

RNAP

Mfd(R953A), ATP

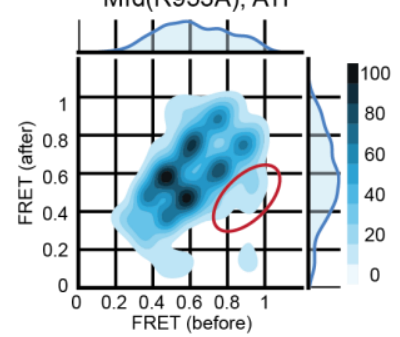

B RNAP, ATP $\gamma \mathrm{S}(0.5 \mathrm{mM})$
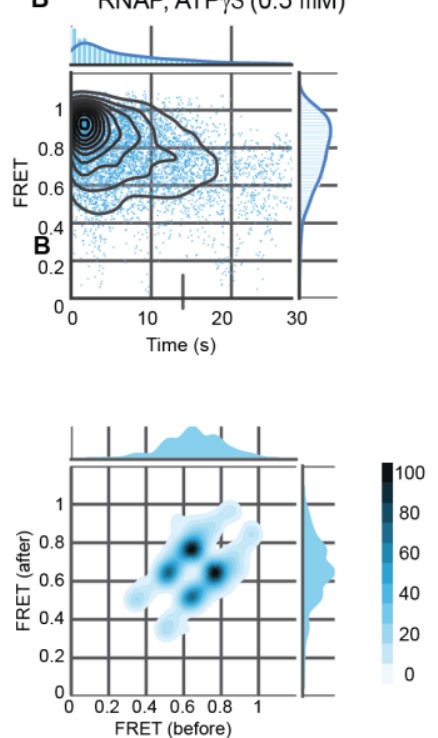
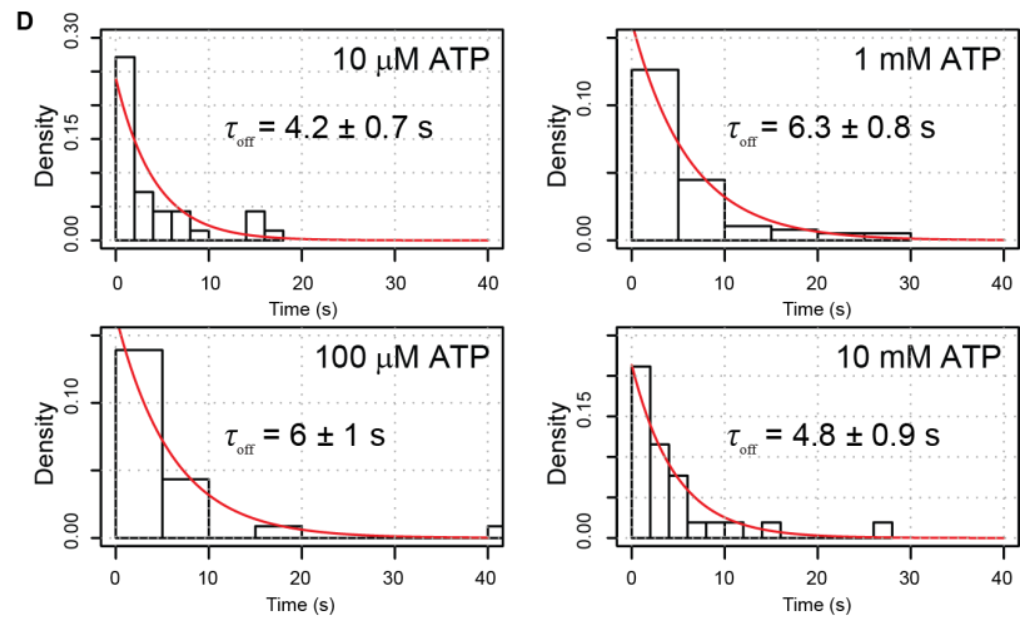

E RNAP, ATP $(0.1 \mu \mathrm{M})$
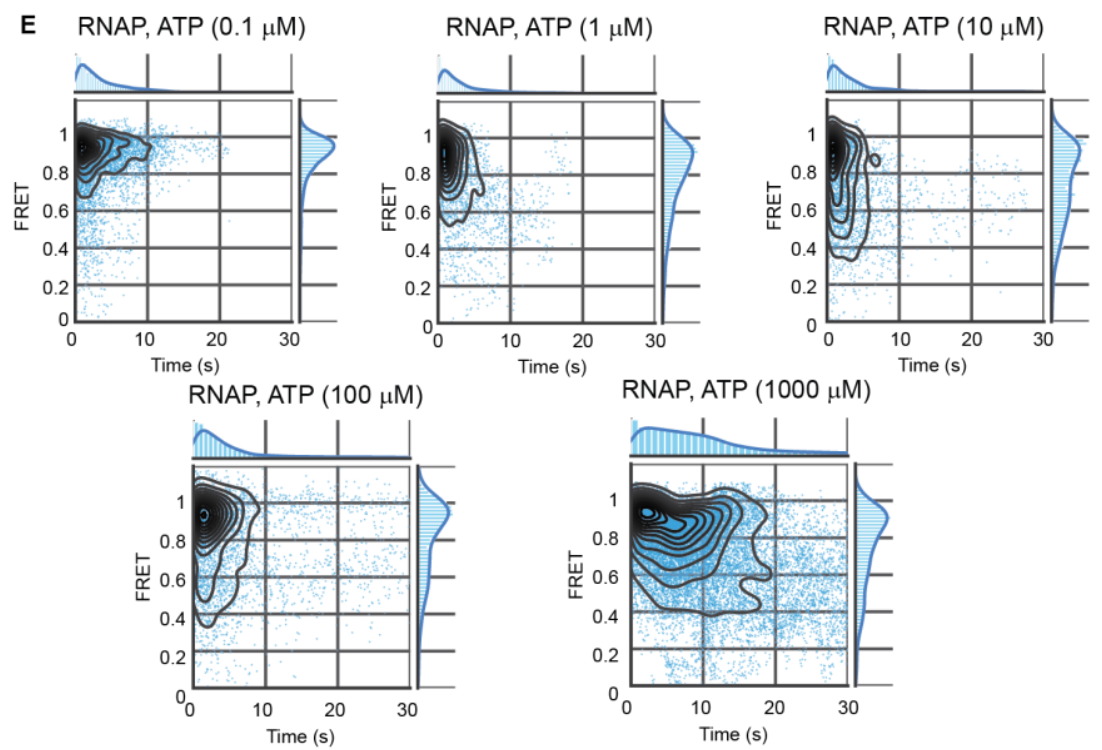
Figure 4

A

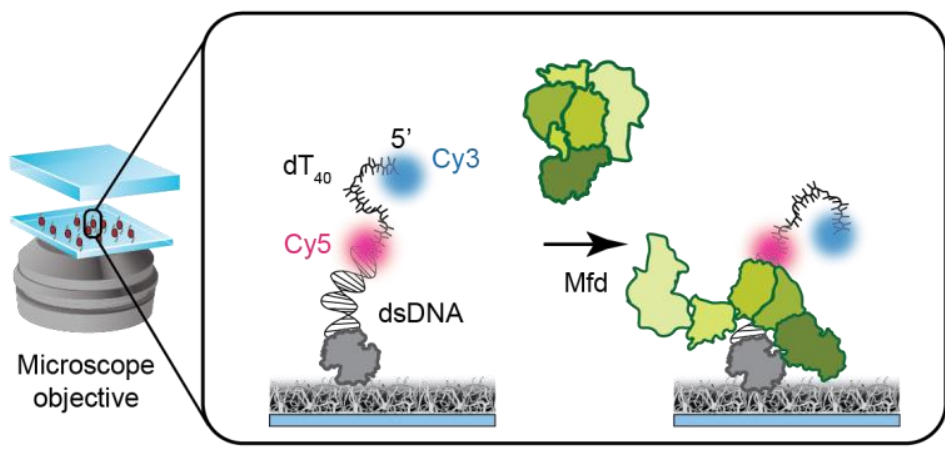

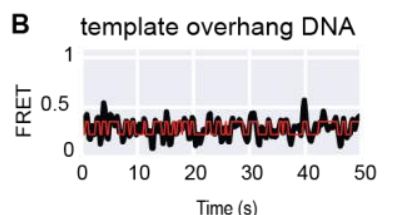

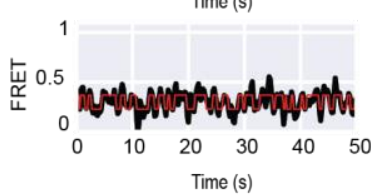

C

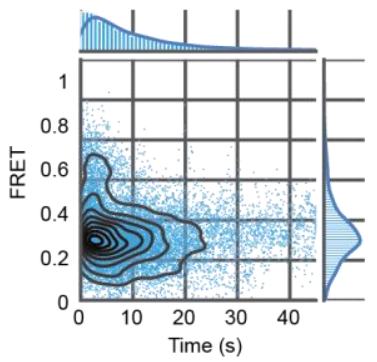

D Mfd, ATP $\gamma \mathrm{S}$

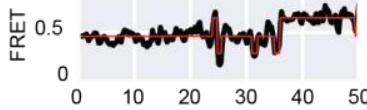

Time (s)

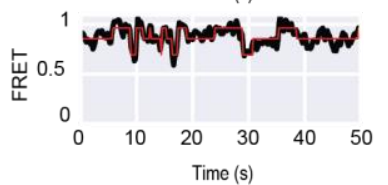

E

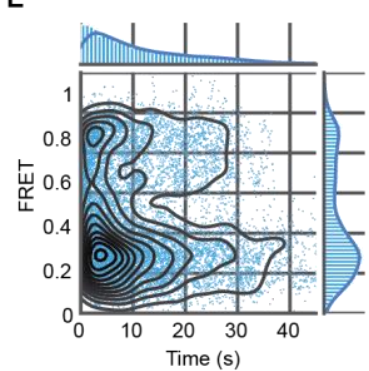

F

Mfd, ATP

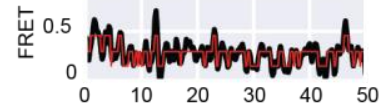

Time (s)
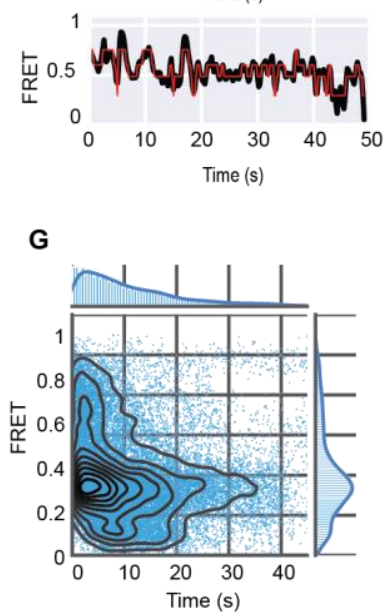
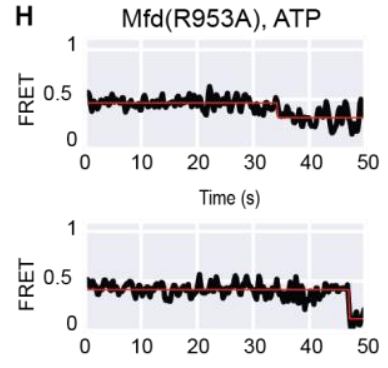

Time (s)

I

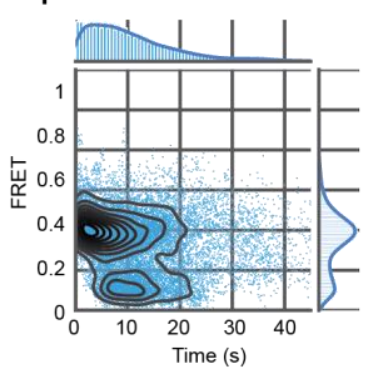


bioRxiv preprint doi: https://doi org/10.1101/2021.04.02.438179; this version posted April 2, 2021. The copyright holder for this preprint (which was not certified by peer review) is the author/funder. All rights reserved. No reuse allowed without permission.

\section{Supplemental Figure 4}
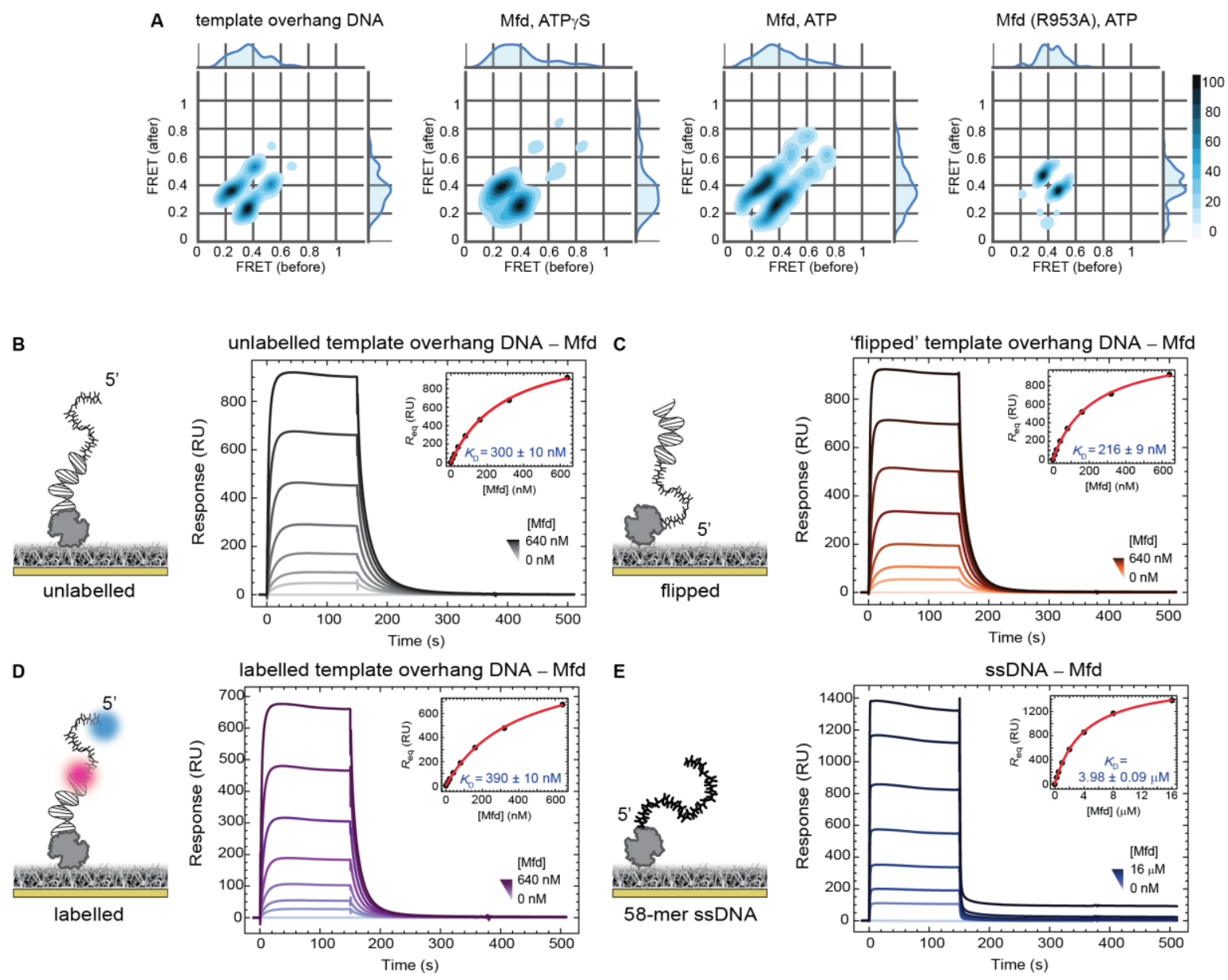

F

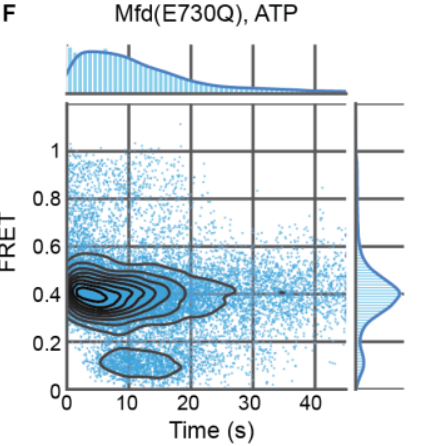

H
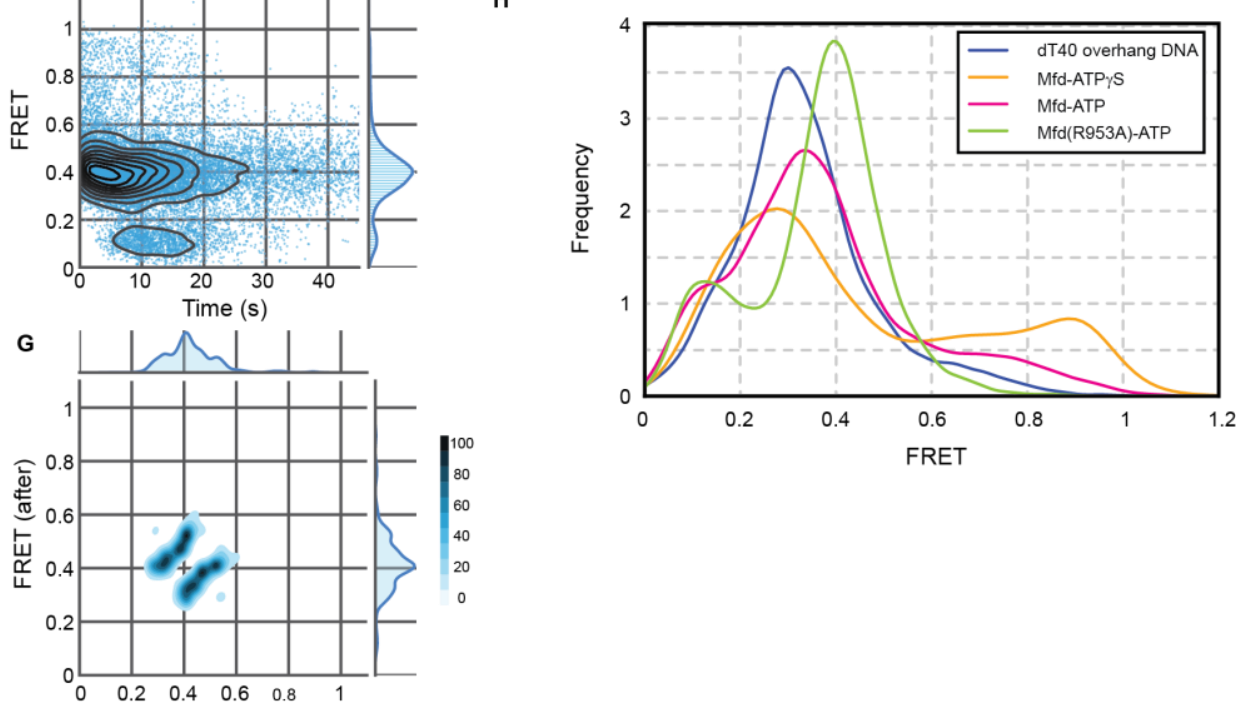
bioRxiv preprint doi: https://doi.org/10.1101/2021.04.02.438179; this version posted April 2, 2021. The copyright holder for this preprint (which was not certified by peer review) is the author/funder. All rights reserved. No reuse allowed without permission.

\section{Figure 5}

A

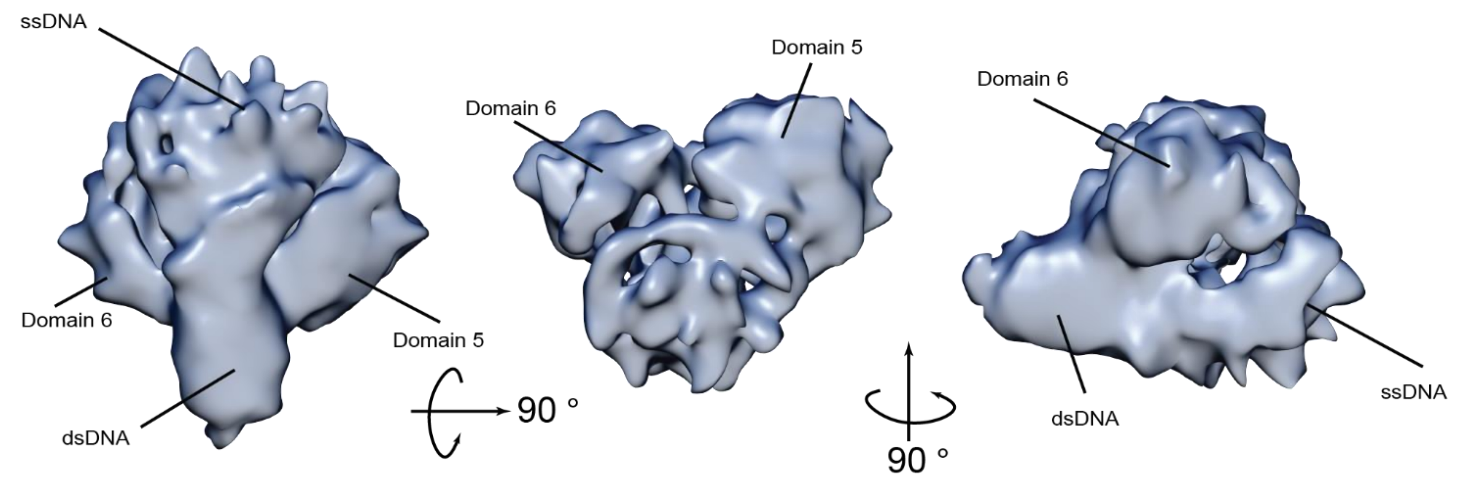

B
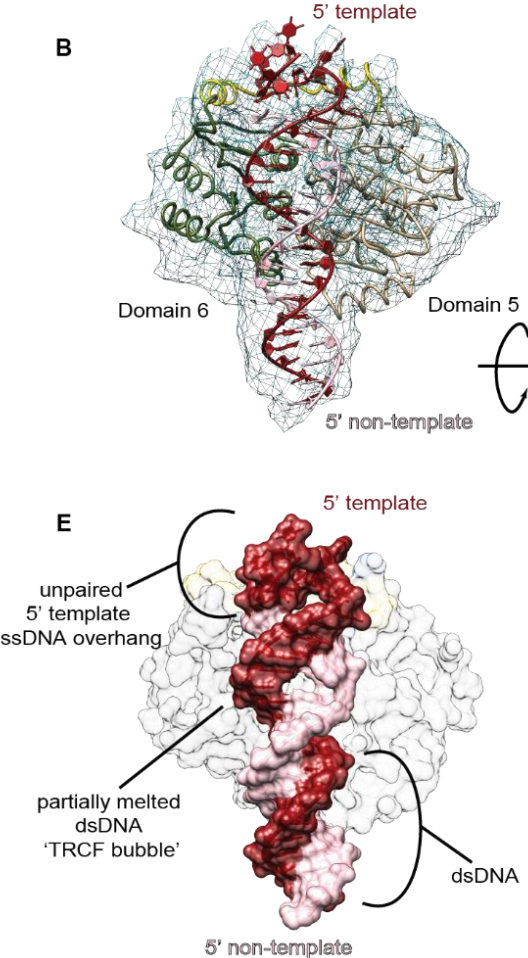

c

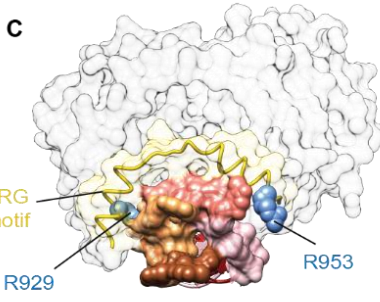

$90^{\circ}$
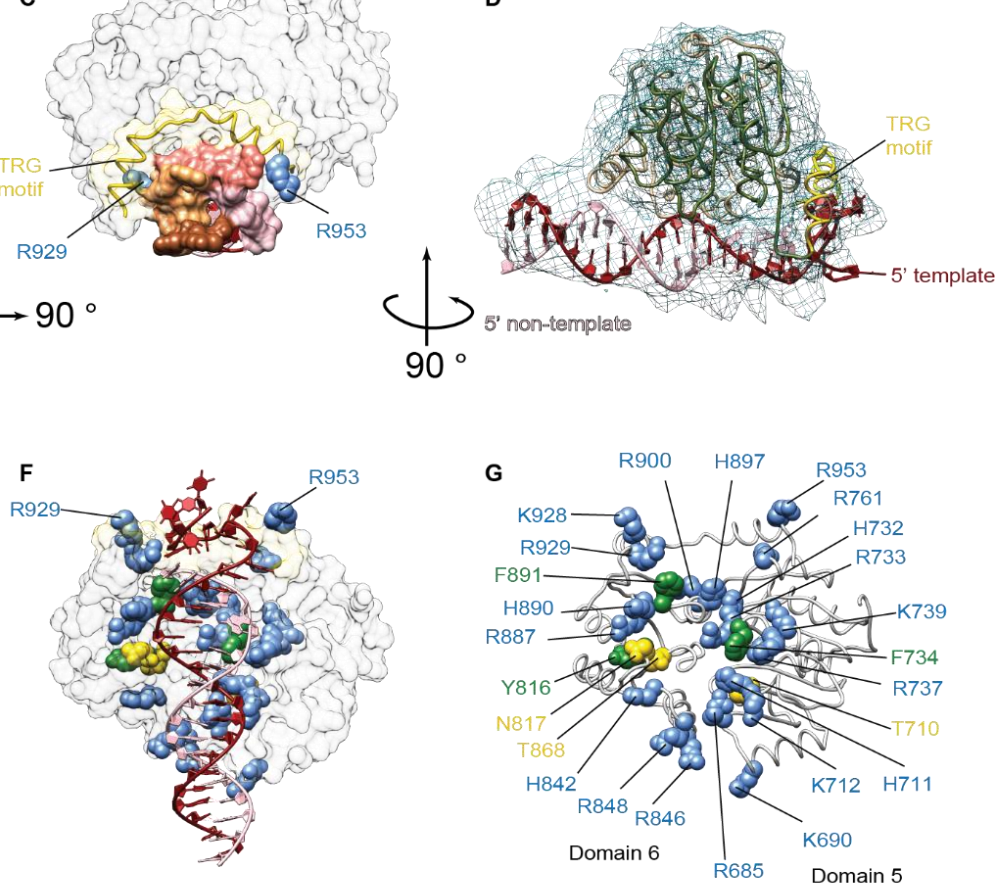
bioRxiv preprint doi: https://doi.org/10.1101/2021.04.02.438179; this version posted April 2, 2021. The copyright holder for this preprint (which was not certified by peer review) is the author/funder. All rights reserved. No reuse allowed without permission.

Supplemental Figure 5
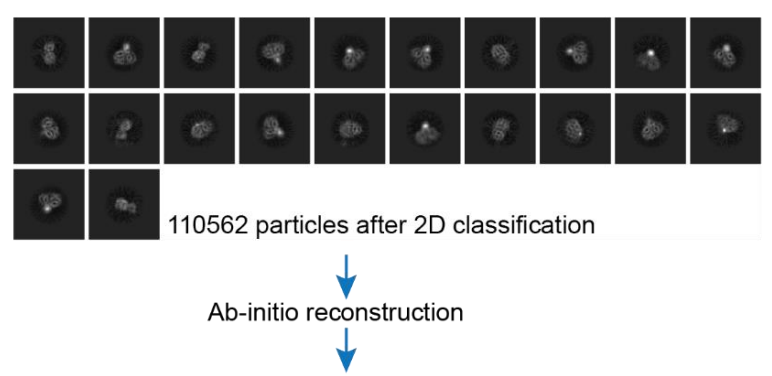

Heterogeneous refinement

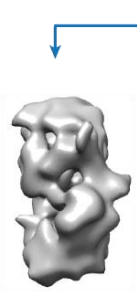

21161 $19 \%$

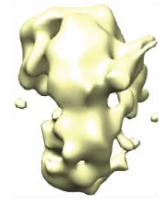

25104 $23 \%$

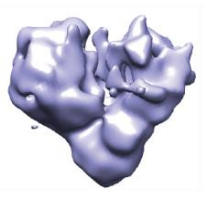

32579

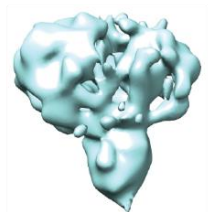

31718

$29 \%$

$\downarrow$ Homogeneous refinement Fourier Shell Correlation

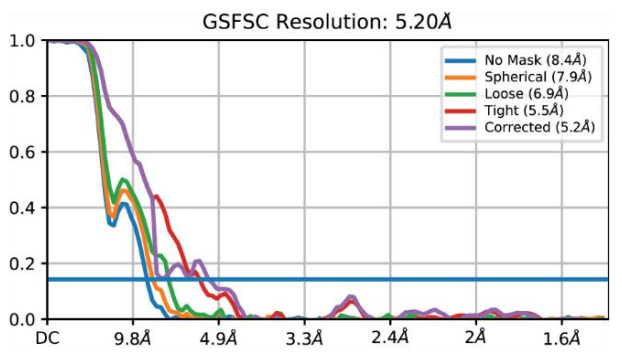

C Angular distribution
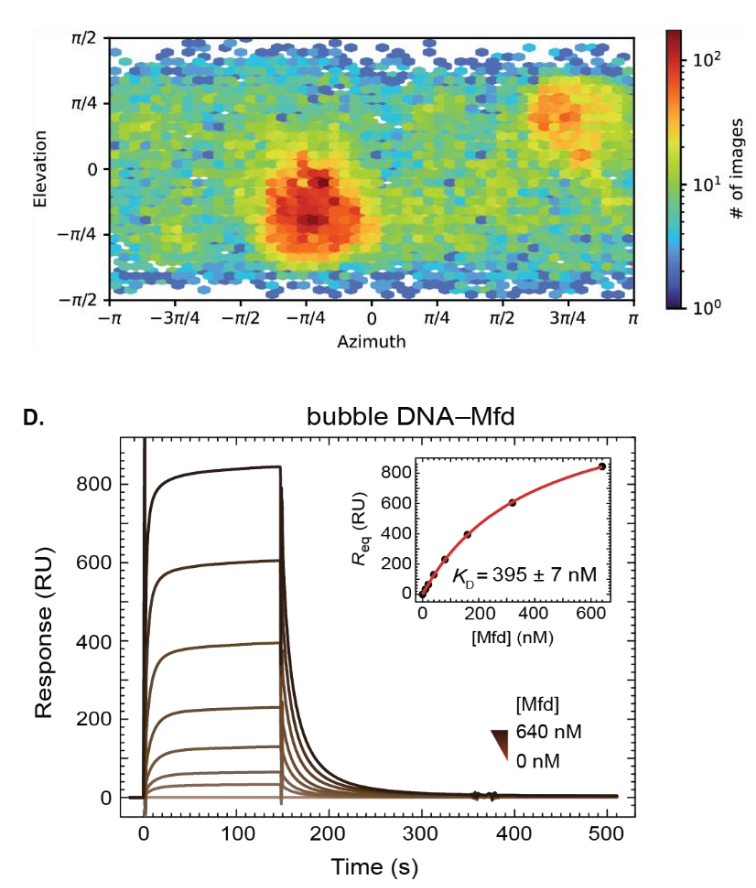

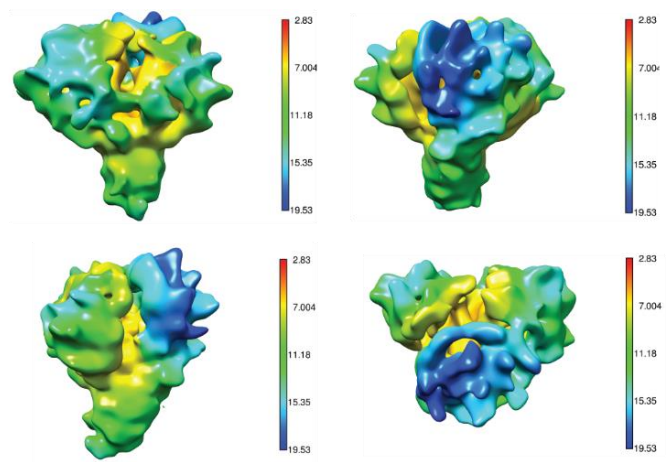

Structure with local resolution 
Figure 6

A

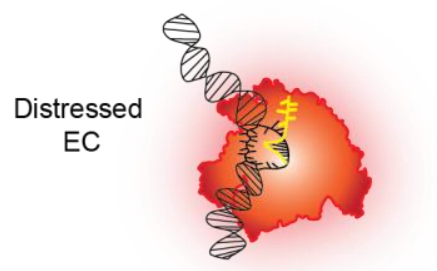

B<smiles>C[Te]C(C)(C)C</smiles>

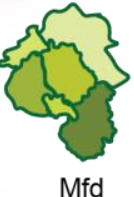

Mfd

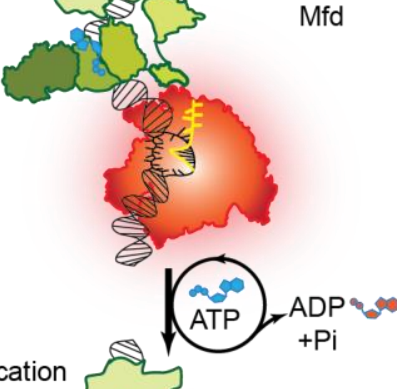

C
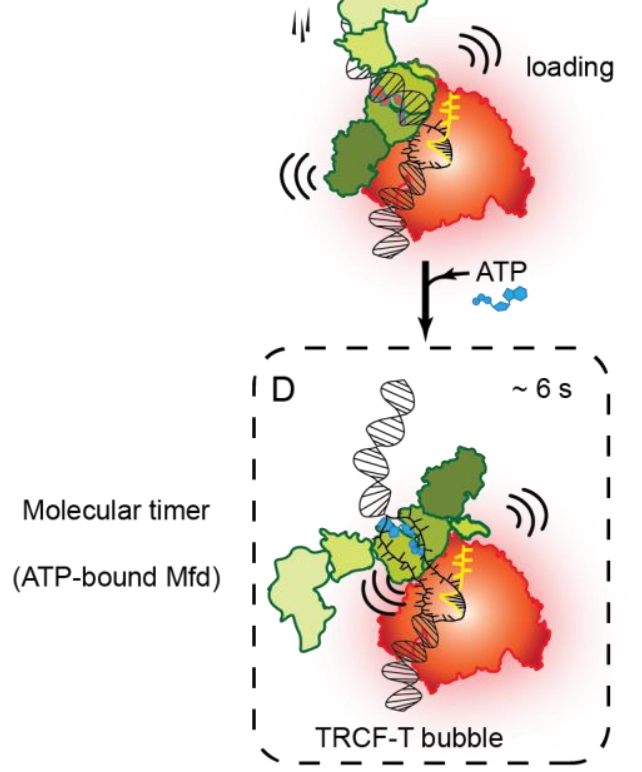

NTP

$\mathrm{PPi}$
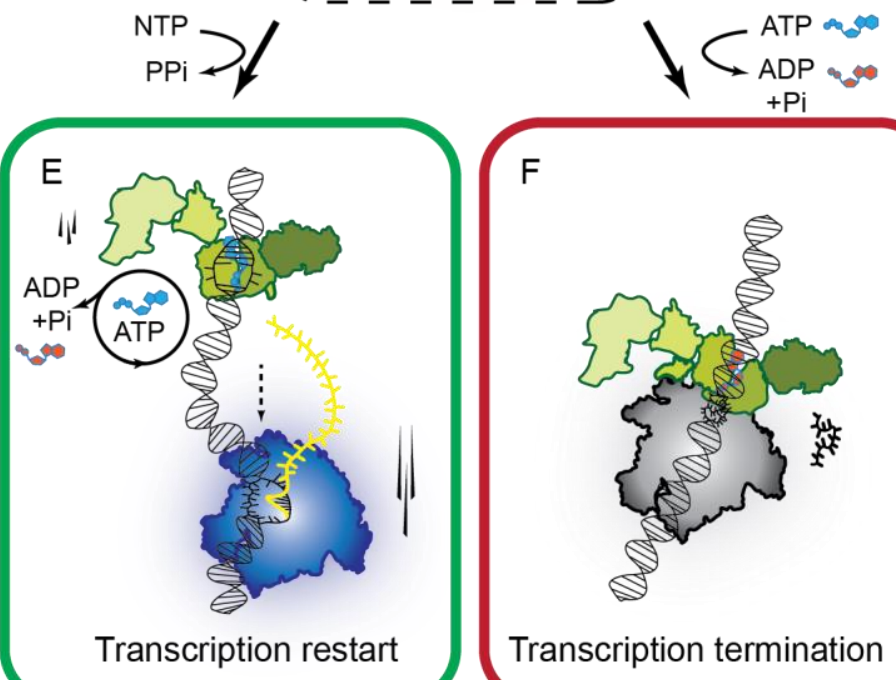

$\mathrm{F}$

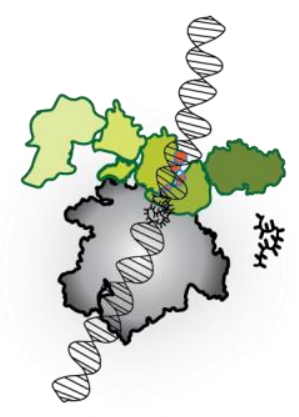

Transcription termination 